\title{
Bidirectional Signaling of Neuregulin-2 Mediates Formation of GABAergic Synapses and Maturation of Glutamatergic Synapses in Newborn Granule Cells of Postnatal Hippocampus
}

\author{
○Kyu-Hee Lee, ${ }^{1}$ Hyunsu Lee, ${ }^{1}$ Che Ho Yang, ${ }^{1}$ Jeong-Soon Ko, ${ }^{1}$ Chang-Hwan Park, ${ }^{2}$ Ran-Sook Woo, ${ }^{3}$ 이oo Yeon Kim, ${ }^{4}$ \\ Woong Sun, ${ }^{4}$ Joung-Hun Kim, ${ }^{5}$ Won-Kyung Ho, ${ }^{1}$ and Suk-Ho Lee ${ }^{1}$ \\ ${ }^{1}$ Cell Physiology Laboratory Department of Physiology, bioMembrane Plasticity Research Center, Neuroscience Research Institute, Seoul National \\ University College of Medicine, Seoul 03080, Korea, ${ }^{2}$ Graduate School of Biomedical Science and Engineering, Hanyang University, Seoul 04763, Korea, \\ ${ }^{3}$ Department of Anatomy and Neuroscience, College of Medicine, Eulji University, Daejeon 34824, Korea, ${ }^{4}$ Department of Anatomy, Korea University \\ College of Medicine, Seoul 02841, Korea, and ${ }^{5}$ Department of Life Sciences, POSTECH, Pohang 37673, Korea
}

Expression of neuregulin-2 (NRG2) is intense in a few regions of the adult brain where neurogenesis persists; however, little is understood about its role in developments of newborn neurons. To study the role of NRG2 in synaptogenesis at different developmental stages, newborn granule cells in rat hippocampal slice cultures were labeled with retrovirus encoding tetracycline-inducible microRNA targeting NRG2 and treated with doxycycline (Dox) at the fourth or seventh postinfection day (dpi). The developmental increase of GABAergic postsynaptic currents (GPSCs) was suppressed by the early Dox treatment ( $4 \mathrm{dpi})$, but not by late treatment $(7 \mathrm{dpi})$. The late Dox treatment was used to study the effect of NRG2 depletion specific to excitatory synaptogenesis. The Dox effect on EPSCs emerged $4 \mathrm{~d}$ after the impairment in dendritic outgrowth became evident (10 dpi). Notably, Dox treatment abolished the developmental increases of AMPA-receptor mediated EPSCs and the AMPA/NMDA ratio, indicating impaired maturation of glutamatergic synapses. In contrast to GPSCs, Dox effects on EPSCs and dendritic growth were independent of ErbB4 and rescued by concurrent overexpression of NRG2 intracellular domain. These results suggest that forward signaling of NRG2 mediates GABAergic synaptogenesis and its reverse signaling contributes to dendritic outgrowth and maturation of glutamatergic synapses.

Key words: dentate gyrus; ErbB4; neuregulin; neurogenesis; synaptogenesis

Significance Statement

The hippocampal dentate gyrus is one of special brain regions where neurogenesis persists throughout adulthood. Synaptogenesis is a critical step for newborn neurons to be integrated into preexisting neural network. Because neuregulin-2 (NRG2), a growth factor, is intensely expressed in these regions, we investigated whether it plays a role in synaptogenesis and dendritic growth. We found that NRG2 has dual roles in the development of newborn neurons. For GABAergic synaptogenesis, the extracellular domain of NRG2 acts as a ligand for a receptor on GABAergic neurons. In contrast, its intracellular domain was essential for dendritic outgrowth and glutamatergic synapse maturation. These results imply that NRG2 may play a critical role in network integration of newborn neurons.

\section{Introduction}

Neuregulins belong to a family of peptide hormones that contains epidermal growth factor (EGF)-like domain. Neuregulin-2 (NRG2) has been identified as a second member of the neuregu-

Received April 24, 2015; revised Nov. 2, 2015; accepted Nov. 9, 2015.

Author contributions: K.-H.L., H.L., and S.-H.L. designed research; K.-H.L., H.L., C.H.Y., J.-S.K., and J.Y.K. performed research;H.L., C.-H.P., and R.-S.W. contributed unpublished reagents/analytic tools; K.-H.L., C.H.Y., R.-S.W., J.Y.K., W.S., J.-H.K., W.-K.H., and S.-H.L. analyzed data; K.-H.L. and S.-H.L. wrote the paper. lins with a domain structure similar to neuregulin-1 (NRG1) (Busfield et al., 1997; Carraway et al., 1997; Chang et al., 1997). Although NRG1 and NRG2 are strongly expressed early in the developing brain, especially in germinal layers (Corfas et al., 
1995; Longart et al., 2004), they are expressed in distinct cell populations in the brain and display distinct subcellular distribution. In the adult brain, the expression of NRG1 becomes low and sporadic but widespread over the brain (Longart et al., 2004; Liu et al., 2011). In contrast, intense expression of NRG2 persists in three regions of an adult brain: the cerebellum, olfactory bulb, and the hippocampal dentate gyrus (Carraway et al., 1997; Longart et al., 2004). NRG2 is preferentially expressed in dendrites, whereas NRG1 is targeted to the axon as well as dendrites (Longart et al., 2004). Such distinct temporal and spatial expression patterns of NRG2 imply that it may have nonredundant biological functions.

Developmental consequences induced by depletion or knockout of the NRG1 gene or exogenous NRG1 injection have been extensively studied over the last decades. Neuregulins can function as a ligand for ErbB4, an EGF receptor. NRG1/ErbB signaling has been implicated in GABAergic circuit formation, axon myelination, and synaptic plasticity (Ghashghaei et al., 2006; Li et al., 2007; Woo et al., 2007; Fazzari et al., 2010; Abe et al., 2011; Ting et al., 2011; Yin et al., 2013). Furthermore, type III NRG1 plays a role in morphological development of cortical neurons independently of ErbB kinase activity (Chen et al., 2008; Chen et al., 2010). Although NRG2/ErbB4 signaling has been implicated in the proliferation of neural stem cells and neuroblasts in the subventricular zone (Ghashghaei et al., 2006), little is understood about whether it plays a role in synaptogenesis of newborn neurons.

In general, GABAergic synaptogenesis precedes glutamatergic synaptogenesis in the early developmental stage of newborn neurons. It has been demonstrated that depolarization induced by GABAergic synaptic inputs in immature neurons is important for AMPA receptor insertion to nascent glutamatergic synapses that express only NMDA receptors (Akerman and Cline, 2006; Wang and Kriegstein, 2008), suggesting that GABAergic synaptogenesis is a prerequisite for maturation of glutamatergic synapses in immature neurons. Given that NRG1/ErbB4 signaling plays a critical role in GABAergic synapse formation (Fazzari et al., 2010), it should be differentiated whether the effects of neuregulin signaling on glutamatergic synapse maturation are direct or secondary to its effect on GABAergic synaptogenesis. Whereas CNS-specific knock-out of ErbB2/4 impaired dendritic spine maturation (Barros et al., 2009), a recent study has shown that conditional knockout of ErbB4 in neocortical pyramidal neurons caused no effect on excitatory synapses (Fazzari et al., 2010). Therefore, cell- and developmental-stage-specific gene manipulation is essential to elucidate biological functions exerted directly by neuregulins.

NRG2 is the major type of neuregulin expressed in hippocampal dentate granule cells (GCs). We set out to address the question of whether NRG2 plays any cell-autonomous role in the development of newborn GCs, especially during two critical developmental periods when GABAergic and glutamatergic synapses are established. To this end, we depleted NRG2 at different developmental stages of newborn GCs using a tetracycline-inducible (Tet-On) expression system (Gossen and Bujard, 1992). Here, we show that NRG2 plays a central role in GABAergic synaptogenesis and glutamatergic synapse maturation in newborn GCs via forward and reverse signaling pathways, respectively.

Correspondence should be addressed to Dr. Suk-Ho Lee, Department of Physiology, Seoul National University College of Medicine, 103 Daehak-ro, Jongno-gu, Seoul 03080, Republic of Korea. E-mail: leesukho@snu.ac.kr. DOI:10.1523/JNEUROSCI.1585-15.2015

Copyright $\odot 2015$ the authors $\quad 0270-6474 / 15 / 3516480-15 \$ 15.00 / 0$

\section{Materials and Methods}

Organotypic slice culture. All experiments were performed with the approval of the animal experiment ethics committee at the Seoul National University College of Medicine. The entorhino-hippocampi of postnatal day 6-9 (P6-P9) Sprague Dawley rat pups (Charles River) of either sex were cultured by using previously described membrane interface techniques (Lee et al., 2012b). Briefly, the transverse hippocampal slices (thickness, $300 \mu \mathrm{m}$ ) were obtained with vibratome (DTK-1000 ZERO 1; Dosaka) and carefully placed on the surface of $30 \mathrm{~mm}$ porous $(0.4 \mu \mathrm{m})$ membrane insert (Millicell-CM; Millipore) set in a 6-well plate on $1 \mathrm{ml}$ of culture medium. Because deprivation of major synaptic inputs of GCs may alter the responsiveness to NRG2, we took great care to preserve the perforant pathway and entorhinal cortex in the organotypic culture. The culture medium was a mixture of 50\% minimum essential medium, $25 \%$ horse serum, 24\% Eagle's buffered salt solution, and 1\% penicillin-streptomycin (PS). Glucose was added to reach a final concentration of $36 \mathrm{~mm}$. To examine the role of NRG2 in synaptogenesis, we infected the slices with retrovirus immediately after transferring slices on membrane. After $4 \mathrm{~d}$ in culture, the medium was changed to serum-free Neurobasal medium with $2 \%$ B-27 supplement, $1 \%$ GlutaMAX-I, $1 \%$ PS, and $5 \mathrm{~mm}$ glucose added. Culture medium was changed every other day. Slice cultures were maintained at $36^{\circ} \mathrm{C}$ in a $5 \% \mathrm{CO}_{2}$ humidified incubator. All media and reagents for organotypic culture were purchased from Invitrogen.

Retroviruses, conditional knock-down, and conditional transgene expression. Newborn GCs were labeled using vesicular stomatitis virus glycoprotein pseudotyped recombinant retrovirus encoding EGFP and microRNA. These two genes were cloned downstream of long terminal repeat (LTR) promoter and a Tet-On promoter, respectively, in the same Moloney murine leukemia virus (MMLV)-derived retroviral vector (see Fig. 1C). Therefore, the expression of miR30-based short-hairpin RNA (shRNAmir) was under the control of the tetracycline-inducible expression system (Gossen and Bujard, 1992). We targeted the shRNAmir by inserting the partial sequence of NRG2 (5'-GGTGTGTCACTCGCCTGCTACT- $3^{\prime}$ ) into the backbone of miR30, an endogenous microRNA (Dickins et al., 2005). This will be referred to as "shNRG2mir." The fluorescent protein mCherry-targeting sequence (5'-GAACATCAAGTTGGACATCACC$3^{\prime}$ ) was used for nontargeting control of shRNAmir (shNTmir). Figure $1 C$ shows the schematic diagram for the expression cassette of EGFP, reverse tetracycline transactivator 3 (rtTA3), and shRNAmir. We engineered retroviral construct by inserting this cassette $(2.6 \mathrm{~kb})$ between the LTRs of EGFP-CL retroviral vector (Lee et al., 2012a). For rescue experiments, C-terminal myc-tagged intracellular domain of NRG2 (NRG2-ICD) was cloned downstream of the Tet-On promoter in the TagRFP-CL retroviral vector, where TagRFP was substituted instead of the EGFP in EGFP-CL (see Fig. 7A1). To package retroviruses, each of these plasmid DNAs were transfected into 293GPG packaging cells using Lipofectamine 2000 (Invitrogen). Supernatant containing viral particles was harvested at 3-10 d after transfection and centrifuged at $6000 \times g$ for $16 \mathrm{~h}$ at $4^{\circ} \mathrm{C}$. The viral pellet was then resuspended in $1 / 500$ of the original volume of DPBS (Invitrogen). The dentate gyrus of a hippocampal slice culture was infected with the virus solution $(0.3$ $\mu \mathrm{l})$ within $5 \mathrm{~min}$ after placement of hippocampal slices on the membrane insert as described previously (Namba et al., 2007; Lee et al., 2012a). For conditional knock-down or conditional expression, supplement of doxycycline (Dox, $2 \mu \mathrm{g} / \mathrm{ml}$ ) in culture medium was started $2 \mathrm{~d}$ before the time that knock-down or overexpression was desired.

Single-cell RT-PCR. Hippocampal newborn GCs were labeled by retrovirus encoding EGFP. To obtain total mRNA of newborn GC, wholecell patch-clamp was attained on the EGFP-expressing soma of a dentate GC at 5-6 days postinfection (dpi). Pipette solution was composed of the following (in mM): $140 \mathrm{~K}$-gluconate, 5 di-Tris-phosphocreatin, $5 \mathrm{NaCl}, 4$ MgATP, 0.4 $\mathrm{Na}_{2}$ GTP, 15 HEPES, 2.5 Na-pyruvate, pH 7.3 adjusted with $\mathrm{KOH}$, together with $10 \%$ RNaseOUT (v/v), a recombinant ribonuclease (RNase) inhibitor (Invitrogen). After rupturing the giga-ohm-sealed cell membrane patch, cytosolic contents were sucked into the glass pipette by applying negative pressure. To prevent the pipette solution from being contaminated by RNase, the whole-cell patch mode was converted to outside-out configuration by gently pulling up the pipette away from the 
cell. The pipette tip containing the cytosolic contents was broken into a $0.2 \mathrm{ml}$ PCR tube with $7 \mu$ l of premixture containing $1 \mu \mathrm{l}$ of dNTP $(25 \mathrm{~mm}$ each), $1 \mu \mathrm{l}$ of $0.1 \mathrm{~m}$ DTT, $1 \mu \mathrm{l}$ of random hexamer oligonucleotides as primers, and $4 \mu \mathrm{l}$ of $5 \times$ first-strand buffer (Invitrogen). Then, $1 \mu \mathrm{l}$ of SuperScript III reverse transcriptase $(200 \mathrm{U} / \mu \mathrm{l}$; Invitrogen) and RNasefree water were added to make a total volume of $20 \mu \mathrm{l}$ of solution. All subsequent steps for synthesizing cDNA followed the user manual of SuperScript III reverse transcriptase. Random primers were annealed to mRNA template by incubating at $65^{\circ} \mathrm{C}$ for $5 \mathrm{~min}$. cDNA was synthesized under the following conditions: $25^{\circ} \mathrm{C}$ for $5 \mathrm{~min}$ and $50^{\circ} \mathrm{C}$ for $60 \mathrm{~min}$. The reaction was terminated by heating at $70^{\circ} \mathrm{C}$ for $15 \mathrm{~min}$. Synthesized singlestrand cDNA was amplified by PCR with GoTaq Green Master Mix (Promega) and the following primers: NRG $2 \beta$ forward 5 '-CAGCAGTT CGCAATGGTCAACT-3', reverse $5^{\prime}$-GTAATCTGCCATCTGGATCTCC$3^{\prime} ; \beta$-actin forward 5'-GCCGCATCCTCTTCCTCCCTG-3', reverse 5'GGGGCCGGACTCATCGTACTC-3'. The PCR products were examined by standard agarose gel electrophoresis.

In situ hybridization and immunohistochemistry. For in situ hybridization, a postnatal week 4 (PW4) rat was perfused by PBS and subsequently fixed with $4 \%$ paraformaldehyde (PFA). Brain was postfixed with $4 \%$ PFA and then dipped with $30 \%$ sucrose in PBS. After sinking, the brain was embedded in optimum cutting temperature compound and sectioned serially into $16 \mu \mathrm{m}$ sections. The sequence of the ErbB4 mRNA target probe was manufactured by Advanced Cell Diagnostics. A probe targeting the bacterial gene dapB was used as a negative control. In situ hybridization of ErbB4 mRNA was performed on sectioned samples according to the RNAscope multiplex fluorescence kit. Briefly, tissue sections were dried at $50^{\circ} \mathrm{C}$ for $30 \mathrm{~min}$ and then fixed with $4 \%$ PFA for 15 min; dehydrated by $50 \%, 70 \%$, and $100 \%$ ethanol gradually for 5 min; and pretreated with the probe kit before hybridization. After hybridization with ErbB 4 mRNA at $40^{\circ} \mathrm{C}$ for $2 \mathrm{~h}$, the RNAscope multiplex fluorescence kit was used for signal amplification. Fluorescence detection was performed using Atto 550 (red fluorescence). Immunohistochemistry was performed using a primary antibody against the doublecortin (DCX, 1:500; Santa Cruz Biotechnology) for $1 \mathrm{~h}$ at $37^{\circ} \mathrm{C}$. Alexa Fluor 488conjugated donkey anti-rabbit IgG was applied for $30 \mathrm{~min}$ (1:500) with Hoechst 33343 for staining nuclei. The sections were washed three times and mounted.

Western blotting. HEK293 cells were seeded in $100 \mathrm{~mm}$ culture dishes at $\sim 70 \%$ confluence and transfected with C-terminally myc-tagged NRG2 (NRG2-myc) plus shNRG2mir or plus shNTmir (as control) using the calcium phosphate protocol. Mouse cDNA of NRG2 was purchased from Origene (catalog \#MR217926). After culture for $48 \mathrm{~h}$, the cells were washed twice with PBS ( $\mathrm{pH}$ 7.4) solubilized in ice-cold lysis buffer containing $20 \mathrm{~mm}$ Tris (pH 7.4 adjusted with $\mathrm{HCl}$ ) $140 \mathrm{~mm} \mathrm{NaCl}$, $10 \%$ sucrose, 1 mm EDTA, 1 mm $\mathrm{Na}_{3} \mathrm{VO}_{4}, 1 \% \mathrm{NP}-40$ (v/v), and $0.2 \%$ protease inhibitor mixture. Cell lysates were mixed with equal volume of the $2 \times$ SDS sample buffer and boiled for $5 \mathrm{~min}$. Proteins were separated by SDS-PAGE and transferred to PVDF membranes (Millipore). The resulting blots were blocked for $1 \mathrm{~h}$ in PBS plus $0.1 \%$ Triton X-100 $(0.1 \%$ PBST) containing 5\% skim milk (blocking solution) and incubated overnight at $4^{\circ} \mathrm{C}$ with specific primary antibodies: mouse monoclonal anti-c-myc (1:1000; Cell Signaling Technology) or mouse monoclonal $\operatorname{anti-} \beta$-actin (1:5000; Santa Cruz Biotechnology) as loading controls. Immunoblots were washed with washing buffer containing $1 \% \mathrm{NP}-40$ and $0.1 \%$ SDS in PBS for $1 \mathrm{~h}$, then with PBS twice each for $30 \mathrm{~min}$ at room temperature. Blots were blocked in blocking solution for $30 \mathrm{~min}$ and incubated with horseradish peroxidase-conjugated goat anti-mouse IgG (1:5000; Jackson ImmunoResearch). After washing with washing buffer and PBS, detection was performed using enhanced chemiluminescence reagent (GE Healthcare Bioscience). The membranes were then exposed to x-ray films (Agfa Gevaert).

Immunohistochemistry. Expressions of NRG1 and NRG2 in the hippocampal slice cultures and tetracycline-inducible knock-down of NRG2 or expression of myc-tagged NRG2-ICD were confirmed by immunostaining of endogenous NRG1 and NRG2 or myc in organotypic cultured hippocampal slices. Dox treatment was started 3-5 d before fixation. Slices were fixed overnight at $4^{\circ} \mathrm{C}$ by submersion in $4 \%$ PFA dissolved in PBS. The next day, after 3 washes with PBS containing $0.3 \%$ Triton X-100
(0.3\% PBST), slices were incubated in blocking solution (5\% donkey serum in $0.3 \%$ PBST) for $1 \mathrm{~h}$ at room temperature and then with rabbit polyclonal anti-NRG2 IgG (1:500; Millipore; see Fig. 1E) or mouse monoclonal anti-NRG1 IgG (1:200; Millipore; see Fig. $1 F)$ or mouse monoclonal anti-myc (1:2000; Cell Signaling Technology; see Fig. 7A2) diluted in blocking solution overnight at $4^{\circ} \mathrm{C}$. After 3 washes in $0.3 \%$ PBST for $10 \mathrm{~min}$, slices were incubated with Cy3-conjugated goat antirabbit (1:400; Invitrogen), Alexa Fluor 647-conjugated donkey antimouse (1:200; Jackson ImmunoResearch), or FITC-conjugated donkey anti-mouse (1:200; Jackson ImmunoResearch) diluted in blocking solution for $1 \mathrm{~h}$ at room temperature. Finally, the slices were washed 3 times in $0.3 \%$ PBST store in PBS until confocal imaging was performed.

Electrophysiology. Postsynaptic current (PSC) was recorded in EGFPpositive newborn GCs using whole-cell patch-clamp techniques while perfusing a slice with artificial CSF (aCSF). The aCSF contained the following (in mM): $124 \mathrm{NaCl}, 26 \mathrm{NaHCO}_{3}, 3.2 \mathrm{KCl}, 2.5 \mathrm{CaCl}_{2}, 1.3 \mathrm{MgCl}_{2}$, $1.25 \mathrm{NaH}_{2} \mathrm{PO}_{4}$, and 10 glucose, $\mathrm{pH} 7.4$ adjusted by saturating with carbogen $\left(95 \% \mathrm{O}_{2}, 5 \% \mathrm{CO}_{2}\right)$. PSC was evoked by stimulation $(200 \mu \mathrm{s} \mathrm{du}-$ ration; 1-10 $\mathrm{V}$ intensity) of axons passing through the inner molecular layer (IML) of hippocampal dentate gyrus using a concentric bipolar electrode with $30 \mathrm{~s}$ interstimulus interval. The stimulation electrode was placed at least $200 \mu \mathrm{m}$ apart laterally from the cell under recording to avoid direct stimulation of a recorded cell. To isolate GABAergic PSCs (GPSCs), the membrane potential was held at $0 \mathrm{mV}$, which is the reversal potential of excitatory PSCs (EPSCs). The internal pipette solution for recording GPSCs contained the following (in $\mathrm{mm}$ ): $140 \mathrm{~K}$-gluconate, 5 di-Tris-phosphocreatin, $5 \mathrm{NaCl}, 4 \mathrm{MgATP}, 0.4 \mathrm{Na}_{2} \mathrm{GTP}, 15$ HEPES, 2.5 Na-pyruvate, 1 EGTA, pH 7.3 adjusted with $\mathrm{KOH}$. For isolation of EPSCs, we could not use a $\mathrm{GABA}_{\mathrm{A}}$ receptor blocker because it induces epileptiform activity. Instead, GPSCs were blocked intracellularly using a CsF-based pipette solution containing the following (in mM): $140 \mathrm{CsF}, 2$ $\mathrm{MgCl}_{2}, 10$ HEPES, 1 EGTA (Marchionni and Maccaferri, 2009; Schwartz et al., 2011), and a $\mathrm{Cl}^{-}$channel blocker, $4,4^{\prime}$-diisothiocyanatostilbene2,2'-disulfonic acid (DIDS, 1 mm) (Hollrigel et al., 1998), pH 7.3 adjusted with $\mathrm{CsOH}$. AMPA receptor- and NMDA receptor-mediated EPSCs (AMPA-EPSCs and NMDA-EPSCs) were recorded at holding potentials of -60 and $+40 \mathrm{mV}$, respectively. The amplitudes of GPSCs and AMPAEPSCs were measured at their peak; the amplitude of NMDA-EPSCs was measured at $50 \mathrm{~ms}$ after stimulation to exclude AMPA-EPSC contribution. The paired-pulse ratio (PPR) was calculated from the mean amplitude of the second PSCs divided by the mean amplitude of the first PSCs. The interpulse interval for GPSCs and NMDA-EPSCs was $500 \mathrm{~ms}$ and that of AMPA-EPSCs was $30 \mathrm{~ms}$. The AMPA-to-NMDA EPSC ratio (AMPA/NMDA ratio) was calculated by dividing the peak amplitude of AMPA-EPSCs by the amplitude of NMDA-EPSCs measured at $50 \mathrm{~ms}$, similar to the procedure described by Mierau et al. (2004). To measure monosynaptic GPSCs, monopolar stimulation electrode (a glass pipette filled with aCSF) was placed $\sim 100 \mu \mathrm{m}$ lateral from the recording position. Monosynaptic responses were evoked by extracellular stimulation (200 $\mu$ s duration; $1-25$ V intensity) with 30 s interstimulus interval in the presence of $20 \mu \mathrm{M}$ CNQX and $50 \mu \mathrm{M}$ APV to block glutamatergic transmission. The amplitudes of monosynaptic GPSCs compared in Figures $3 C$ and $6 B$ were obtained from a minimal stimulation protocol. The first criterion for the minimal stimulation is an abrupt appearance of synaptic responses as a gradual increase in the stimulation intensity. There should be a distinct threshold for such all-or-none activation of synaptic responses. Moreover, further increase in the stimulation intensity should not change the synaptic responses by $\sim 20 \%$ increase in stimulus intensity from the threshold (Dvorzhak et al., 2013). Typical voltage intensities required for minimal stimulation ranged between 5 and $12 \mathrm{~V}$.

In vivo experiments. P16-P22 SD rats were anesthetized by inhalation of $\sim 1-4 \%$ isoflurane, shaved, and then placed in a stereotaxic frame (Neurostar; Stoelting). All surgical procedures were performed under aseptic conditions. Retrovirus encoding EGFP plus Tet-On/shNRG2mir was infused through a glass micropipette which was prepared using a vertical puller (Narishige PC-10) and a pipette beveler (beveling angle, $30^{\circ}$; Narishige EG-4). Virus solution of $0.5 \mu \mathrm{l}$ was injected at a rate of 0.15 $\mu \mathrm{l} / \mathrm{min}$ by a $10 \mu \mathrm{l}$ Hamilton syringe attached to a digital microinjector (Stoelting). The injection coordinates were $-4.8 \mathrm{~mm} \mathrm{~A} / \mathrm{P}, \pm 4.4 \mathrm{~mm}$ 
$\mathrm{M} / \mathrm{L},-4.6 \mathrm{~mm} \mathrm{D} / \mathrm{V}$ from the bregma. The glass pipette was slowly removed after holding it in the injection site for $3 \mathrm{~min}$ to allow for the viral diffusion. After suturing, the rats were returned to the cages and lidocaine/antibiotics ointment was applied over the sutured region. Rats were given drinking water containing $2 \mathrm{mg} / \mathrm{ml}$ Dox plus $2 \%$ sucrose (Dox-containing water) or $2 \%$ sucrose alone (Dox-free water) from $5 \mathrm{dpi}$ or from 9 dpi. The drinking water was changed every other day. Acute hippocampal slices (thickness, $300 \mu \mathrm{m}$ ) were prepared from 12-21 dpi (P28-P40) rats as described in Lee et al. (2007).

Drugs. All chemicals were purchased from Sigma-Aldrich except CNQX and bicuculline methiodide, which were from Tocris Bioscience; APV, which was from Abcam; and AG-1478, which was from Calbiochem. Stock solutions were stored at $-20^{\circ} \mathrm{C}$ and diluted to the desired concentration in aCSF just before use. Anti-c-erbB4 Ab-3 mouse monoclonal antibody (MS-304) was purchased from Thermo Scientific.

Sholl analysis and measurement of total dendritic length. EGFP fluorescence expressed in newborn GCs were imaged using a confocal laser scanning microscope (FV300; Olympus) and a $40 \times$ water-immersion objective. For analysis of dendritic morphology, 3D reconstructions were made using the Simple Neurite Tracer plugin in the ImageJ-based image processing package Fiji (Longair et al., 2011). The dendritic complexity and length were quantified by Sholl analysis plugin and tracing algorithms in NeuronJ plugin, respectively. For Sholl analysis, the concentric circles were drawn every $10 \mu \mathrm{m}$ from the first concentric circle, which had a diameter $5 \mu \mathrm{m}$ encircling the soma, and the numbers of branch intersections were counted in each circle.

Data analysis. Data were analyzed using Igor-Pro (version 6.1; WaveMetrics) or IBM SPSS statistics version 21.0 software. Statistical data are expressed as mean \pm SEM and $n$ indicates the number of cells studied. If not mentioned otherwise, the statistical significance of differences between two experimental conditions was evaluated using Student's $t$ test. Comparisons between input-output curves of synaptic currents and between results of Sholl analysis were analyzed using repeated-measures (RM) one-way ANOVA (see Figs. 3A1, 4A, 5B, 7D2). To compare between cumulative lengths of neurites, the KolmogorovSmirnov (K-S) test was used (see Figs. 5C, 7D3). Differences were considered as significant when $p<0.05$ (n.s. indicates no statistical significance; $\left.{ }^{\star} p<0.05 ;{ }^{\star *} p<0.01 ;{ }^{* *} p<0.001\right)$.

\section{Results}

\section{Confirmation of NRG2 expression in newborn GCs and} conditional knock-down strategy of NRG2

Although NRG2 is highly expressed in the GC layer of dentate gyrus of adult hippocampus (Anton et al., 2004; Longart et al., 2004), it is not clear whether newborn GCs express NRG2. To address this question, newborn GCs from cultured rat hippocampal slice were labeled with EGFP-expressing retrovirus and then their mRNA was analyzed by single-cell RT-PCR (see Materials and Methods for details). There are two alternative splicing isoforms of NRG2, $\alpha$ and $\beta$, and only the $\beta$ isoform is capable of stimulating ErbB4 (Hobbs et al., 2002). The cytoplasm of EGFPpositive single newborn GC was aspirated using a whole-cell patch pipette (Fig. 1A1) and mRNA therein was amplified using a primer pair specific for an ErbB4-stimulating isoform, NRG2 $\beta$. NRG $2 \beta$ transcript was detected in 7 of 8 newborn GCs and $\beta$-actin, used as positive control, was detected in all samples (Fig. 1A2).

We further examined the expressions of NRG1 and NRG2 in hippocampal slice cultures at 7 and 14 dpi (equivalent to PW2 and PW3 in vivo, respectively) using immunohistochemistry. Consistent with Longart et al. (2004), the NRG1 immunosignal was weaker in the dentate gyrus compared with the CA1 and CA3 regions (Fig. 1B1), suggesting that NRG2 may be a major ligand for ErbB4 in the dentate gyrus. Next, we investigated whether newborn GCs express the NRG2 receptor ErbB4. It has been noted that ErbB4 is expressed exclusively in GABAergic interneu- rons, at least in the neocortex and hippocampus (Vullhorst et al., 2009; Fazzari et al., 2010). Nevertheless, the expression of ErbB4 in newborn GCs remains unknown. To determine whether immature GCs express ErbB4, we doubly stained the hippocampus for ErbB4 transcripts and DCX. The former was stained by in situ hybridization and the latter, which is a marker of immature GCs (Rao and Shetty, 2004), was stained by immunohistochemistry. This double staining revealed that the signal of ErbB4 transcripts is noise level in most DCX-immunoreactive cells of the dentate gyrus $(0.59 \pm 0.14, n=180,3$ rats, Fig. $1 B 2)$. In contrast, some cells scattered in the dentate gyrus showed significantly many ErbB4 fluorescent spots $(44.49 \pm 3.17, n=45)$ that were presumably inhibitory interneurons. Therefore, it is unlikely that NRG2 on newborn GCs act as an autocrine ligand.

To study the role of NRG2 in GABAergic and glutamatergic synaptogenensis on newborn GCs, we tried to knock down the NRG2 transcripts specifically in newborn GC using retrovirus. Because NRG2/ErbB4 signaling regulates the proliferation of neuroblasts in the subventricular zone (Ghashghaei et al., 2006), constitutive knock-down of NRG2 may suppress the proliferation of neural progenitor cells in the dentate gyrus. Therefore, we designed a cassette encoding shNRG2mir under the control of the Tet-On promoter (Fig. 1C; see Materials and Methods for details; Gossen and Bujard, 1992) and inserted it into the MMLV retroviral vector. This retroviral construct enabled us to control the period of NRG2 knock-down by treatment with Dox $(2 \mu \mathrm{g} /$ $\mathrm{ml})$. In addition, the cassette encodes the EGFP gene downstream of the constitutively active LTR promoter, so we could label newborn GCs infected by the retrovirus regardless of Dox treatment (Fig. 1C).

The knock-down efficiency of shNRG2mir was tested in HEK293 cells heterologously expressing NRG2-myc together with shNRG2mir or shNTmir. When the HEK293 cells were treated with Dox $(2 \mu \mathrm{g} / \mathrm{ml})$ for $48 \mathrm{~h}$, shNRG2mir, but not shNTmir, almost depleted NRG2-myc. In untreated cells, neither shNTmir nor shNRG2mir depleted NRG2-myc (Fig. 1D). Next, to test whether shNRG2mir efficiently depletes endogenous NRG2 in newborn GCs, we compared immunofluorescence of NRG2 in EGFP-positive newborn GCs at 7 dpi grown in the rat hippocampal slice cultures under control conditions and those treated with Dox from 4 dpi. The immunoreactivity of NRG2 was significantly lower in the Dox-treated newborn GCs compared with the untreated GCs (Fig. $1 E$, untreated, $940.97 \pm 79.87, n=$ 9 vs Dox-treated, $558.60 \pm 21.22, n=27, p<0.001$ ), whereas NRG1 immunoreactivity was not affected by shNRG2mir (Fig. $1 F)$. Repeating the same experiments for newborn GCs at $14 \mathrm{dpi}$ that were untreated or treated with Dox from $10 \mathrm{dpi}$, we obtained similar results (Fig. 1E2, untreated, $822.69 \pm 53.33, n=11$ vs Dox-treated, $466.55 \pm 35.32, n=22, p<0.001)$, indicating that shNRG2mir efficiently depletes endogenous NRG2 in newborn GCs and that knock-down can be induced by Dox treatment.

\section{Developmental changes of postsynaptic currents in newborn GCs under control conditions}

Before studying the effect of NRG2 depletion, we examined the normal developmental increase in the PSC of newborn GCs. Newborn GCs grown in rat hippocampal slice cultures were identified by expression of EGFP (Fig. 2A). At different dpi's, the IML of the hippocampal dentate gyrus was stimulated to evoke GPSCs, AMPAEPSCs, and NMDA-EPSCs (Fig. 2B). The K-gluconate-based pipette solution containing $5 \mathrm{mM} \mathrm{Cl}^{-}$was used for recordings of GPSCs. In hippocampal dentate gyrus, glutamatergic afferent fibers, such as perforant pathways and hilar mossy cell axons, are mostly 
A1

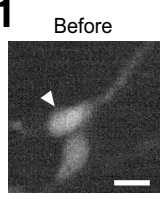

B1
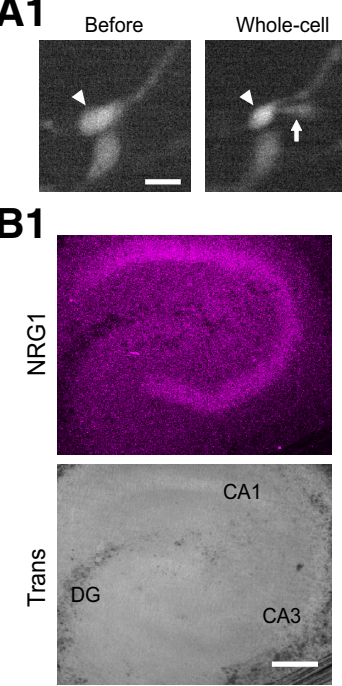

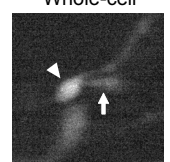

B2
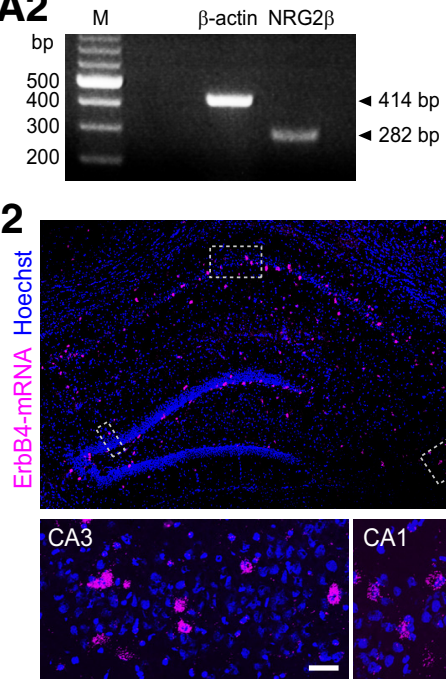

E2
C

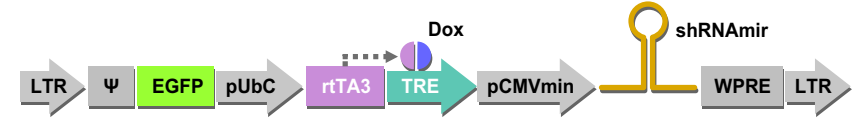

E1 7 dpi

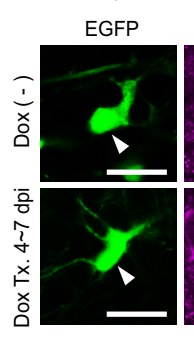

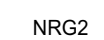

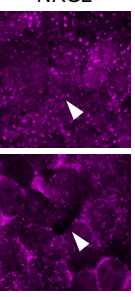

Merge

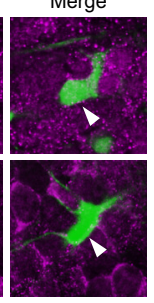

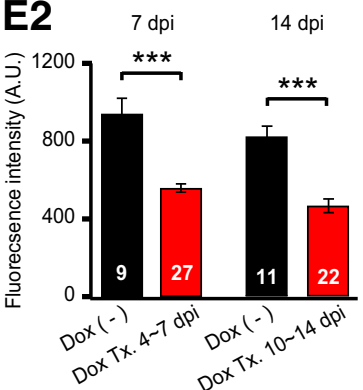

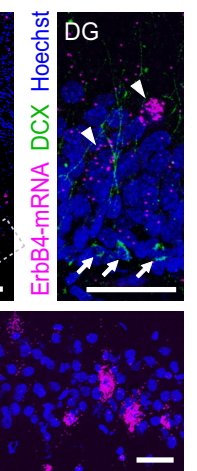

F1 7 dpi EGFP

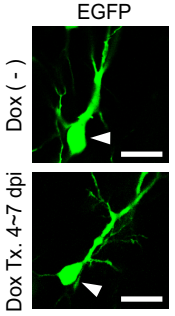

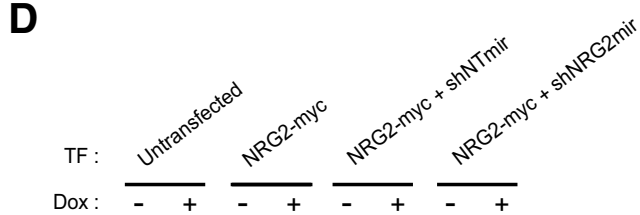

NRG2-myc

$\beta$-actin $-\infty$
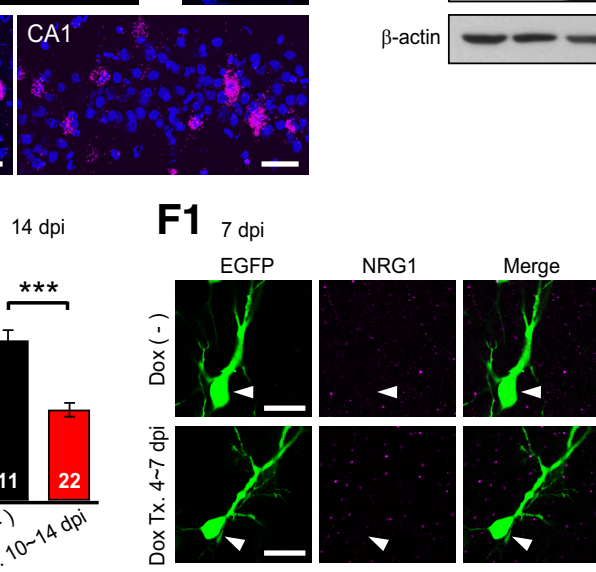

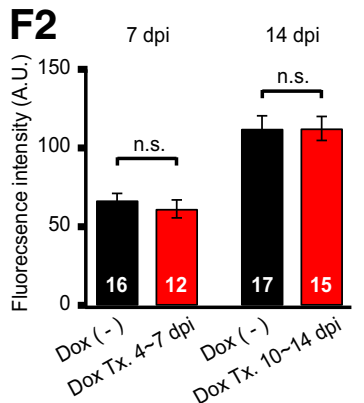

Figure 1. Tests for expression of NRG1, NRG2, and ErbB4 in hippocampal newborn GCs and for shRNAmir-mediated depletion of NRG2. A1, Extraction of cytoplasm for single-cell RT-PCR. Left, Retrovirus-infected newborn GCs were identified by EGFP fluorescence at 5- 6 dpi. Right, Cytoplasm was sucked into the whole-cell pipette with negative pressure. Arrowheads, EGFP-positive newborn GC; arrow, patch pipette. Scale bar, $10 \mu \mathrm{m}$. A2, Representative example for single-cell RT-PCR. Bands for NRG2 $\beta$ and $\beta$-actin (as a positive control) were detected at the predicted size of 282 and $414 \mathrm{bp}$, respectively. The leftmost lane is a size marker. B1, Immunostaining of endogenous NRG1 in the hippocampal slice culture (7 dpi) shows that NRG1 signal is weaker in dentate gyrus (DG) than in CA1 and CA3 regions (top). Bottom, Corresponding DIC image. Scale bar, $300 \mu \mathrm{m}$. B2, Double staining of ErbB4 transcripts (magenta) and DCX (green). Boxed regions of DG, CA3, and CA1 in top left panel are magnified images. ErbB4 signal was negative in the $D C X^{+}$cells (arrow) in the DG. Note that there are many ErbB4 fluorescent spots in $D C X^{-}$ErbB ${ }^{+}$cells (arrowhead). Hoechst33342 (blue) was used for nuclear counterstaining. Scale bar, $40 \mu \mathrm{m}$. C, Schematic of the tetracycline-inducible shRNAmir expression cassette. EGFP and rtTA3 are constitutively expressed under the control of promoters, the $5^{\prime} \mathrm{LTR}$, and human ubiquitin-C promoter (pUbiC), respectively. The rtTA3 binds to TRE and activates the transcription of shRNAmir in the presence of doxycycline. $\Psi$, Packaging signal; rtTA3, reverse tetracycline transactivator; TRE, tetracycline response element; pCMVmin, mini-CMV promoter; WPRE, wood-chuck hepatitis B posttranscriptional regulatory element. D, shNRG2mir was cotransfected with NRG2-myc into HEK293 cells. When Dox ( $2 \mu \mathrm{g} / \mathrm{ml}, 48 \mathrm{~h}$ ) was applied to HEK293 cells, shNRG2mir almost depleted NRG2-myc, but shNTmir did not. The blot of $\beta$-actin is shown as a loading control. TF, Transfection. E1, F1, NRG2 and NRG1 immunofluorescence (magenta) of the dentate GC layer (7 dpi) in organotypic cultures infected with retrovirus encoding EGFP plus Tet-On/shNRG2mir. Slice cultures were treated (TX.) with Dox started at $4 \mathrm{dpi}$ (bottom) or untreated (top) and then fixed and immunostained at $7 \mathrm{dpi}$. Somata of EGFP-positive GCs are indicated by arrowheads. Scale bar, $20 \mu \mathrm{m}$. E2, F2, Mean values for the NRG2 and NRG1 immunofluorescence intensity in the EGFP-positive regions of Dox-treated and untreated slices were quantified at 7 or 14 dpi. Dox treatment was from $4-7$ dpi or from $10-14$ dpi, respectively.

confined to the molecular layer and run in parallel with the GC layer (Buckmaster et al., 1992; Witter, 2007; Chancey et al., 2014), whereas GABAergic axon fibers are highly branched and convoluted over all three layers of dentate gyrus (Soriano and Frotscher, 1989; Bartos et al., 2001; Markwardt et al., 2011). Because a bipolar stimulation electrode was placed at least $200 \mu \mathrm{m}$ laterally apart from the recorded newborn GC, the electrode is more likely to stimulate glutamatergic afferent fibers rather than GABAergic ones. Consistently, putative GPSCs, which were recorded as an outward PSC at the reversal potential of EPSCs $(0 \mathrm{mV})$, were inhibited not only by bicuculline $(20 \mu \mathrm{M}), \mathrm{a} \mathrm{GABA}_{\mathrm{A}}$ receptor blocker, but also by CNQX (20 $\mu \mathrm{M}$ ), an AMPA receptor blocker (Fig. $2 C$ ), implying that the GPSCs evoked by IML stimulation are caused by bisynaptic feedforward GABAergic inputs (Fig. 2B).

AMPA-EPSCs and NMDA-EPSCs were recorded using a CsFbased pipette solution containing $1 \mathrm{~mm}$ DIDS to block $\mathrm{Cl}^{-}$current (see Materials and Methods for details). AMPA-EPSCs were evoked at a holding potential of $-60 \mathrm{mV}$ and completely atten- uated by CNQX (20 $\mu \mathrm{M})$ (Fig. 2D1). NMDA-EPSCs were evoked at a holding potential of $+40 \mathrm{mV}$ and inhibited by $50 \mu \mathrm{M} \mathrm{APV}$, an NMDA receptor blocker (Fig. 2D2). The input-output curves for the three kinds of PSCs in newborn GCs were obtained by plotting the amplitudes of evoked PSCs as a function of stimulus intensity. Figure $2 E$ shows mean input-output curves of GPSCs, AMAP-EPSCs, and NMDA-EPSCs at different dpi's of newborn GCs. Because most of these curves reached a plateau at the stimulation voltage of 6 or $7 \mathrm{~V}$, the mean values of GSPCs and EPSCs at $10 \mathrm{~V}$ were collected and plotted as a function of the age of newborn GCs denoted as dpi (Fig. 2F). This plot represents the normal time courses for the developmental increases of synaptic inputs to newborn GCs grown in slice cultures. The evoked GPSCs distinctly appeared in newborn GCs as early as 6 dpi and their amplitude was steeply increased until $10 \mathrm{dpi}$. After $10 \mathrm{dpi}$, the evoked GPSC amplitude did not further increase. Conversely, AMPA-EPSCs were negligible until $11 \mathrm{dpi}$ and then continuously increased at least to the oldest age we studied ( $~ 16 \mathrm{dpi})$. NMDA- 
A

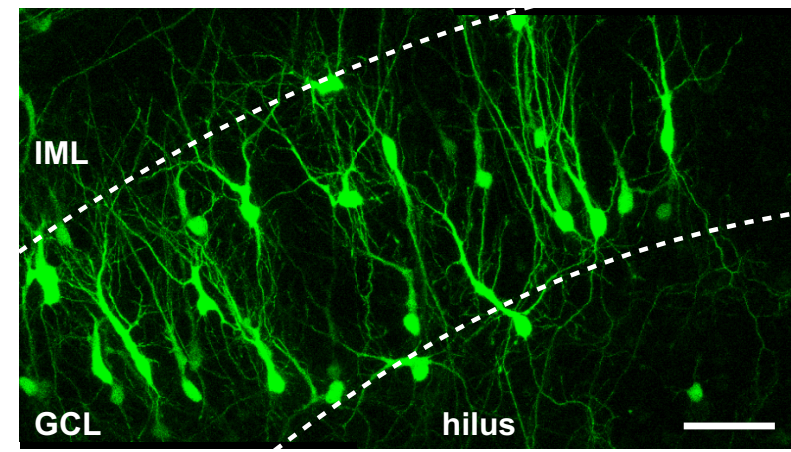

B

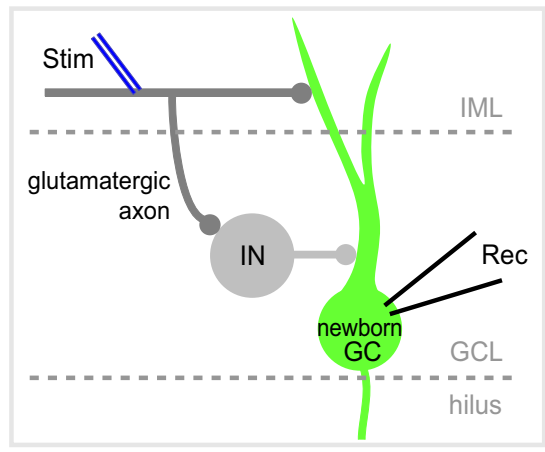

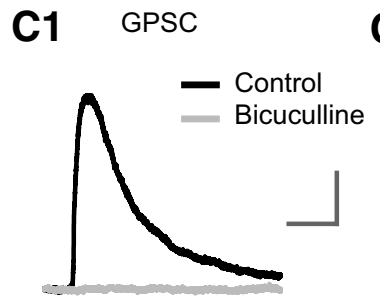

C2
2 GPSC

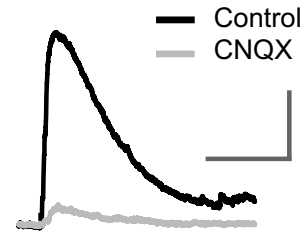

(n)
D1 AMPA-EPSC

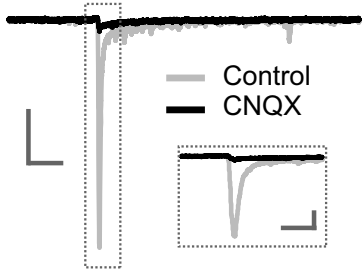

D2 NMDA-EPSC

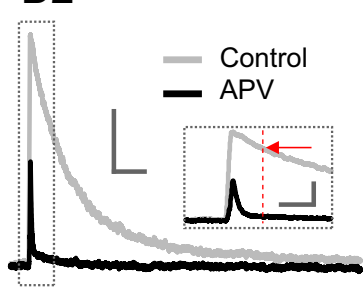

E
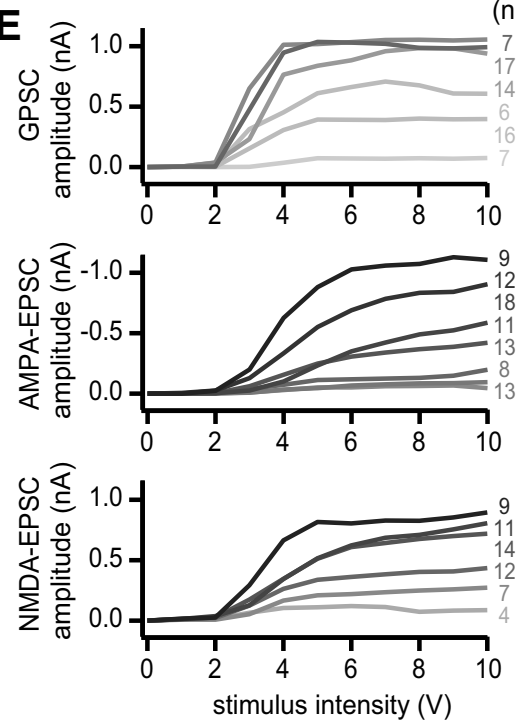

F

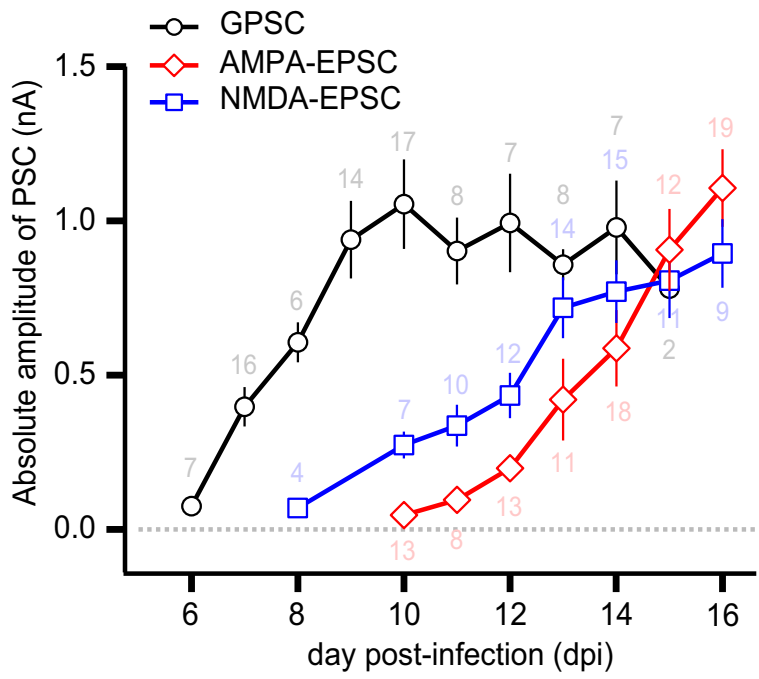

Figure 2. Developmental changes of GPSCs and EPSCs in newborn GCS. A, Dentate gyrus of a hippocampal organotypic slice culture at 10 dpi. Newborn GCs infected by retroviruses expressed EGFP. The borders between the $\mathrm{GC}$ layer $(\mathrm{GCL})$ and hilus or between the $\mathrm{GCL}$ and the IML are marked by dashed lines. Scale bar, $50 \mu \mathrm{m}$. $\boldsymbol{B}$, Schema for excitatory and feedforward inhibitory synapses innervating a newborn GC. Stimulating electrode was located in IML (blue double lines). GPSCs or EPSCs were recorded from EGFP-positive newborn GCs in GCL using whole-cell patch-clamp techniques. Stim, Stimulation electrode; Rec, recording pipette; IN, interneuron. C, Representative GPSCs recorded in newborn GCS. Note that feedforward GPSCs were blocked not only by bicuculline

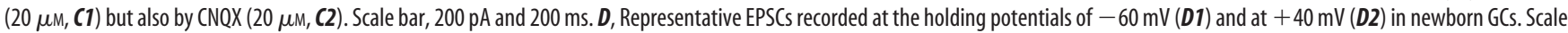
bar, $500 \mathrm{pA}$ and $200 \mathrm{~ms}$. D1, AMPA-EPSCs were abolished by CNQX (20 $\mu \mathrm{M})$. D2, When APV (50 $\mu \mathrm{M})$ was bath-applied to the slice, NMDA-EPSCs were and the outward AMPA-EPSCs were unaffected. The amplitude of NMDA-EPSCs was measured $50 \mathrm{~ms}$ after the stimulation (red dashed line and arrow in the inset). Insets, Traces in an expanded time scale. Scale bars, $500 \mathrm{pA}$ and $50 \mathrm{~ms}$. E, Averaged input- output curves of GPSCs (top), AMPA-EPSCs (middle), and NMDA-EPSCs (bottom) from newborn GCs. The amplitudes of evoked GABAergic or glutamatergic PSCs were plotted as a function of stimulus intensity at different dpi's. F, Developmental changes in amplitudes of GPSCS, AMPA-EPSCS, and NMDA-EPSCs recorded in newborn GCs grown in organotypic slice cultures under control conditions. All PSCs were evoked by stimulation of IML at $10 \mathrm{~V}$. The age of GCs are shown as dpi, which represents the number of days elapsed from the day when the slice was infected by EGFP-encoding retrovirus. The number on each symbol indicates number of cells studied $(n)$.

EPSCs were observed earlier than AMPA-EPSCs and increased gradually. Because the increase rate of NMDA-EPSCs was less steep than that of AMPA-EPSCs, the AMPA/NMDA ratio increased developmentally (see Figs. 2F, 4C).

\section{Expression of NRG2 is essential for GABAergic synapse} formation but dispensable for maintenance in newborn GCs We studied whether depletion of NRG2 has any effect on the developmental changes of GPSCs. Figure $1 E$ shows that NRG2 is depleted to $\sim 50 \%$ in the $3 \mathrm{~d}$ after starting Dox treatment. There- fore, we started to treat cultured hippocampal slices with Dox from 4 dpi and maintained the Dox treatment until the day of the experiment to deplete NRG2 during the GPSC-increasing phase at 6-9 dpi. We obtained input-output curves for mean amplitudes of GPSCs recorded from control and Dox-treated newborn GCs at different dpi's. Typically, GPSCs started to be evoked by stimulation of IML at $3 \mathrm{~V}$ and their amplitude became steady at 6 $\mathrm{V}$ and higher stimulation voltages. Amplitudes of GPSCs were significantly lower in Dox-treated newborn GCs than those in control conditions at both ages (Fig. 3A1; control vs Dox, $F_{(1,35)}$ 

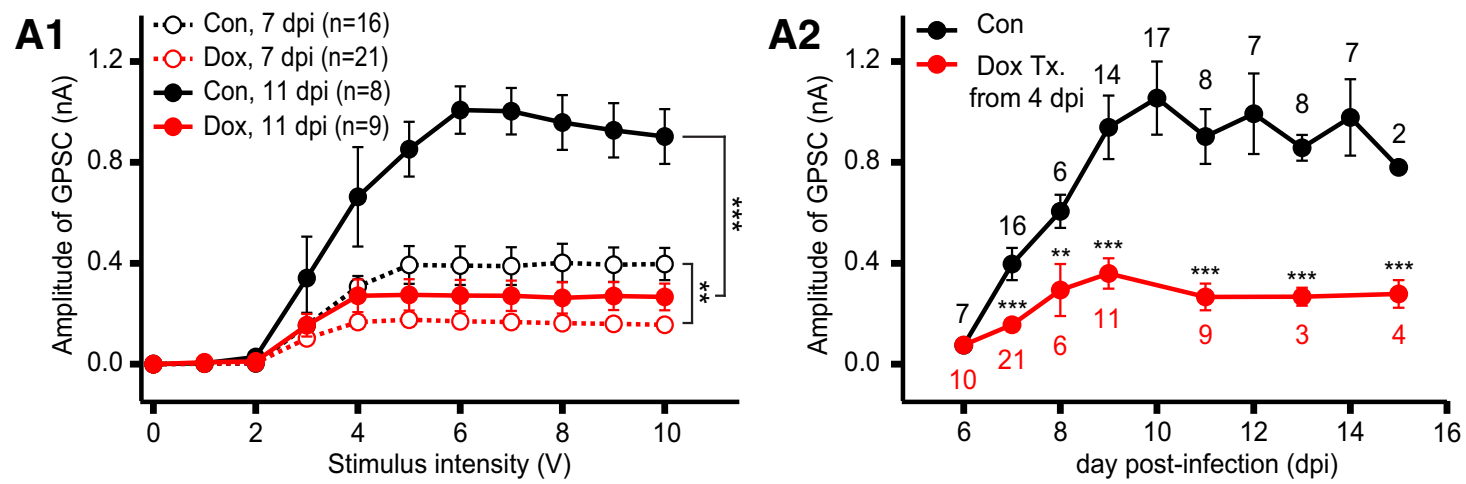

A3

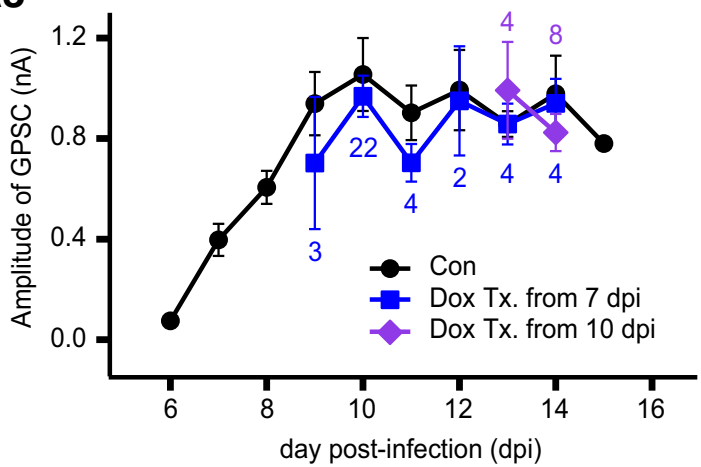

A4

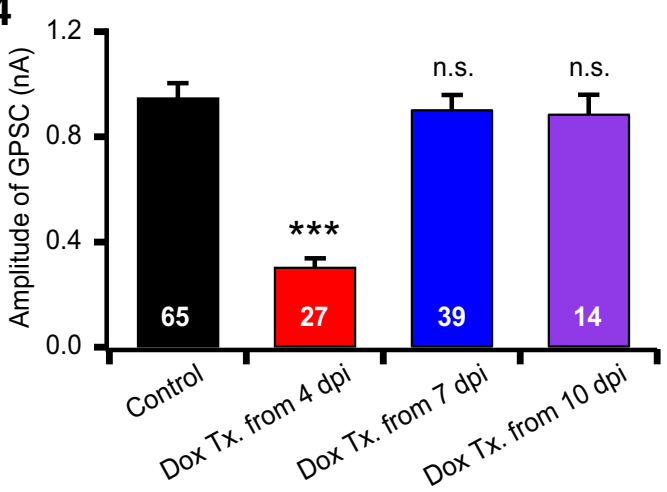

B1 Monosynaptic GPSC

B2

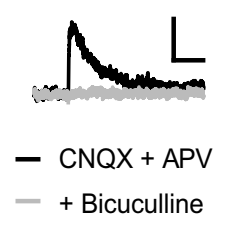

B3

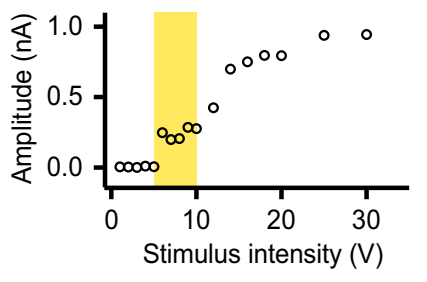

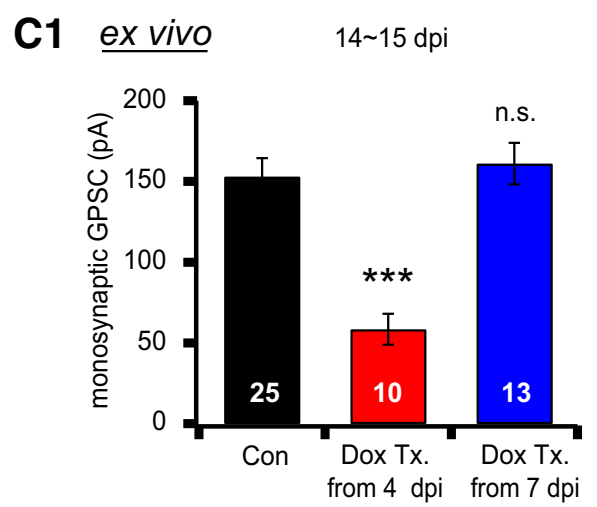
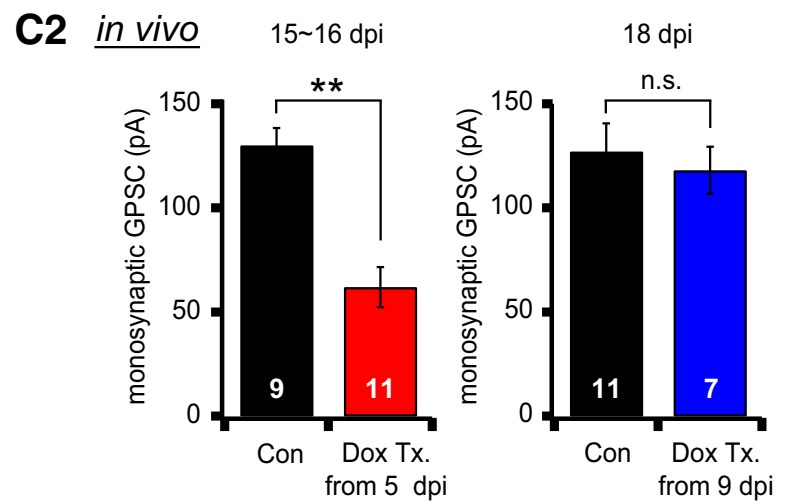

Figure 3. NRG2 is essential for GABAergic synapse formation in newborn GCS. A1, Averaged input- output curves of GPSCs in control (black) and NRG2-depleted (red, Dox-treated from 4 dpi) newborn GCs at $7 \mathrm{dpi}$ (open circles, dotted line) or at $11 \mathrm{dpi}$ (solid circles, solid line). For both GPSCs at 7 and $11 \mathrm{dpi}$, amplitudes were significantly lower in Dox-treated GCs (control vs Dox; $7 \mathrm{dpi}$, $F_{(1,35)}$ $=12.03, p=0.001 ; 11 \mathrm{dpi}, F_{(1,15)}=24.04, p<0.001$ by RM one-way ANOVA). A2, Effects of NRG2 knock-down on the developmental changes of feedforward GPSCs in newborn G(S. To deplete NRG2 hippocampal slices were treated (TX.) with Dox from 4 dpi. GPSCs in Dox-treated GCs were significantly lower than control cells as early as $7 \mathrm{dpi}$. A3, Effects of NRG2 knock-down on GPSCs after the critical period of GABAergic synapse formation (6-9 dpi). The slice cultures were treated with Dox from $7 \mathrm{dpi}$ (blue) or $10 \mathrm{dpi}$ (purple). GPSCs of the treated slice were not significantly different from the untreated control slices at all ages ( $p>0.1$ ). In $\mathbf{A 2}$ and $\mathbf{A 3}$, mean amplitudes of GPSCs evoked by $10 \mathrm{~V}$ stimulation of IML were plotted as a function of dpi. $A 4$, Mean amplitudes of GPSCs in newborn GCs older than $9 \mathrm{dpi}$, at which GPSCs reached a plateau under different Dox treatment conditions. B1, Monosynaptic GPSCS were evoked by minimal stimulation in the presence of AMPA and NMDA receptor antagonists using a monopolar electrode positioned 30-100 $\mu \mathrm{m}$ apart from the soma of a newborn GC. Rec, Recording pipette; IN, interneuron. B2, Representative trace of monosynaptic GPSCs, which was completely blocked by $20 \mu \mathrm{m}$ bicuculline, a GABA $A_{A}$ receptor blocker. Scale bar, $50 \mathrm{pA}$ and $100 \mathrm{~ms}$. B3, Representative input- output curve for monosynaptic GPSCs in newborn GCS. As the stimulation intensity was gradually increased from $1 \mathrm{~V}$, the synaptic responses were all-or-none at a distinct threshold ( $6 \mathrm{~V}$ ) and were kept stable for further increase by $5 \mathrm{~V}$ from the threshold. Such stable range of stimulation intensity was adopted as the voltage intensity for minimal stimulation (marked with yellow). C, Mean amplitudes of monosynaptic GPSCs in newborn GCs grown in slice cultures (ex vivo, (1) or acute slices (in vivo, (2). The starting dates for Dox treatment or feeding (Dox Tx.) are indicated in the abscissa. The recording dates are indicated above the bar graphs. Monosynaptic GPSCs were suppressed by early Dox Tx (from 4 or $5 \mathrm{dpi}$ ), but not by late Dox Tx (from 7 or $9 \mathrm{dpi}$ ), in both ex vivo and in vivo experiments. 
$=12.03, p=0.001$ at $7 \mathrm{dpi} ; F_{(1,15)}=24.04, p<0.001$ at $11 \mathrm{dpi}$, RM one-way ANOVA). The effect of NRG2 depletion on GPSCs is summarized in Figure 3A2, which displays the mean amplitude of GPSCs evoked at $10 \mathrm{~V}$ as a function of dpi. This plot demonstrates that the depletion of NRG2 significantly suppresses the developmental increase of GPSCs.

The above results indicate that NRG2 is essential for developmental increase in GABAergic inputs at 6-9 dpi, which may be a critical period for GABAergic synapse formation under our experimental conditions (Fig. $2 F$ ). To address the question of whether NRG2 is necessary after this critical period, the hippocampal slice cultures were treated with Dox from $10 \mathrm{dpi}$ and GPSCs in the Dox-treated newborn GCs were examined at 13 or 14 dpi. As shown in Figure 3A3, Dox treatment at this age had no significant effect on GPSC amplitudes, arguing against a role of NRG2 in maintenance of GABAergic synapses (control vs Doxtreated from $10 \mathrm{dpi}, p>0.1$ ). To confirm this notion, we started the Dox treatment earlier. The Dox treatment starting at $7 \mathrm{dpi}$ might be sufficient for depleting NRG2 just after the critical period ( 9 or $10 \mathrm{dpi}$ ) because knock-down of NRG2 occurs, at the latest, 2 or $3 \mathrm{~d}$ after starting Dox treatment (Fig. $1 E$ ). This earlier start of Dox treatment also had no significant effect on GPSCs measured in newborn GCs between 9 and 14 dpi (Fig. 3A3, control vs Dox-treated from $7 \mathrm{dpi}, p>0.1$ ). Figure $3 A 4$ shows the age dependence of NRG2 knock-down effects on the GPSCs. Mean values of GPSC amplitudes were measured in newborn GCs older than 9 dpi, when GPSC amplitudes reached a plateau. Dox treatment from $4 \mathrm{dpi}$, but not from 7 or $10 \mathrm{dpi}$, significantly reduced the GPSC amplitude (Fig. 3A4, control, $0.950 \pm 0.054$ $\mathrm{nA}, n=65$; Dox-treated from 4 dpi, $0.306 \pm 0.032 \mathrm{nA}, n=27$, $p<0.001$; Dox-treated from $7 \mathrm{dpi}, 0.905 \pm 0.054 \mathrm{nA}, n=39, p=$ 0.577; Dox-treated from $10 \mathrm{dpi}, 0.889 \pm 0.071 \mathrm{nA}, n=14, p=$ $0.611)$. These results demonstrate that NRG2 is necessary for GABAergic synapse formation during the critical period (6-9 dpi), but, after this critical period, it is dispensable for the maintenance of GABAergic synapses in newborn GCs.

Next, we verified the above notions in monosynaptic GPSCs in slice cultures (ex vivo) and in vivo. Monosynaptic GPSCs were evoked by local stimulation of GC layer $\sim 70 \mu \mathrm{m}$ apart from the recorded cell in the presence of AMPA and NMDA receptor blockers in the bath solution (Fig. 3B1) and recorded as outward PSCs at the holding potential of $0 \mathrm{mV}$. This outward PSC was completely abolished by bicuculline, indicative of monosynaptic GPSCs (Fig. 3B2). A minimal stimulation protocol was used to evoke monosynaptic GPSCs (Fig. 3B3, see Materials and Methods for details). Consistent with the results of feedforward GPSCs, the monosynaptic GPSCs recorded at 14 or 15 dpi ex vivo were suppressed by early Dox treatment, but not by late Dox treatment (Fig. 3C1). For in vivo experiments, newborn GCs were labeled by stereotaxic injection of the same retrovirus used in ex vivo experiments into the hippocampal dentate gyrus of rats (16-22 d old). We studied monosynaptic GPSCs in the acute slice of such rats after feeding them with Dox-free or Dox-containing $(2 \mathrm{mg} / \mathrm{ml})$ water. Because maturation of newborn GCs is faster at a younger age (Overstreet-Wadiche et al., 2006a), Dox feeding started 1 or $2 \mathrm{~d}$ later than ex vivo, that is, at 5 and $9 \mathrm{dpi}$. The amplitude of GPSCs recorded $10 \mathrm{~d}$ later were significantly suppressed by Dox feeding from $5 \mathrm{dpi}$, but not by Dox feeding from $9 \mathrm{dpi}$, confirming the results of ex vivo experiments (Fig. 3C2).
NRG2 is essential for functional maturation of glutamatergic synapses and dendritic growth in newborn GCs

During the early postnatal week, GABAergic inputs exert depolarizing influences on newborn neurons. Perturbation of such GABA-induced depolarization by overexpression of KCC2 or knock-down of NKCC1 blocks normal development of AMPA receptor-mediated neurotransmission (Akerman and Cline, 2006; Ge et al., 2006), suggesting that GABAergic synaptic activity may play a permissive role in the development of glutamatergic synapses. To study the direct roles of NRG2 in the development of glutamatergic synapses, depletion of NRG2 should start after the critical period in order not to interfere with the GABAergic synapse formation. To this end, we started to treat the slice cultures with Dox at $7 \mathrm{dpi}$ (referred to as late Dox treatment), which had no significant effect on GPSCs (Fig. 3A3,A4). Figure $4 A$ shows input-output curves for mean amplitudes of AMPAEPSCs and NMDA-EPSCs evoked by stimulation of IML at 12 and 15 dpi of newborn GCs grown in control or Dox-treated slices. At 12 dpi, neither AMPA-EPSC nor NMDA-EPSC amplitudes were significantly affected by NRG2 knock-down (Fig. 4A1, AMPA-EPSC, $F_{(1,16)}=2.17, p=0.160$; Fig. $4 A 2$, NMDA-EPSC, $F_{(1,17)}=2.33, p=0.145$, RM one-way ANOVA). However, at 15 dpi, both amplitudes were significantly lower in the Dox-treated newborn GCs (Fig. 4A1, AMPA-EPSC, $F_{(1,16)}=15.2$, $p<0.001$; Fig. $4 A 2$, NMDA-EPSC, $F_{(1,17)}=6.78, p=0.019$, RM one-way ANOVA). The late Dox treatment completely suppressed the developmental increase in AMPA-EPSCs and partially suppressed the NMDA-EPSCs to $\sim 40 \%$ of the control (Fig. $4 A, C 1$ ). These findings are summarized in Figure $4 B$, which displays developmental changes of the mean amplitudes for AMPA-EPSCs and NMDA-EPSCs evoked by $10 \mathrm{~V}$ stimulation at control and Doxtreated newborn GCs. The differential effects of NRG2 knockdown on the AMPA-EPSCs and NMDA-EPSCs are more clearly demonstrated by comparing the AMPA/NMDA ratio of control and Dox-treated newborn GCs. AMPA/NMDA ratio was estimated from AMPA-EPSCs and NMDA-EPSCs that were measured in the same cell and evoked by the same stimulation intensity $(10 \mathrm{~V})$. Developmental increase of the AMPA/NMDA ratio in newborn GCs was abolished by knock-down of NRG2 (Fig. 4C2). Because nascent glutamatergic synapses express only NMDA receptors and then AMPA receptors are added as they are matured, the AMPA/NMDA ratio is an index for maturity of glutamatergic synapses (Wu et al., 1996; Akerman and Cline, 2006; Haas et al., 2006). These results indicate that NRG2 is essential for the maturation of glutamatergic synapses in newborn GCs.

To test whether NRG2 knock-down has any effect on dendritic growth, we compared the morphology of newborn GCs in hippocampal slices at different dpi's under control or late Dox treatment conditions (Fig. 5A). The complexity of dendritic arbor was quantified using Sholl analysis (see Materials and Methods for details). The complexity and the total length of dendritic arbors in the Dox-treated GCs were significantly lower than the control GCs, not only at $14 \mathrm{dpi}$, but also at $10 \mathrm{dpi}$ (Fig. 5B, Scholl analysis, $p<0.001$ at both dpi's, RM one-way ANOVA; Fig. $5 C$, cumulative distributions of total dendritic length, $p<0.01$, K-S test). The developmental increase of mean values for total dendritic length was significantly retarded in the Dox-treated GCs (Fig. 5D). Considering that NMDA-EPSCs in the Dox-treated GCs were not significantly different from the control GCs until 13 dpi (Fig. 4B2), the effect of Dox on the dendritic growth emerged $4 \mathrm{~d}$ earlier than that on EPSCs. Because the Dox-induced impairment was specific to dendritic development between 10 and 13 
AMPA-EPSC

A1

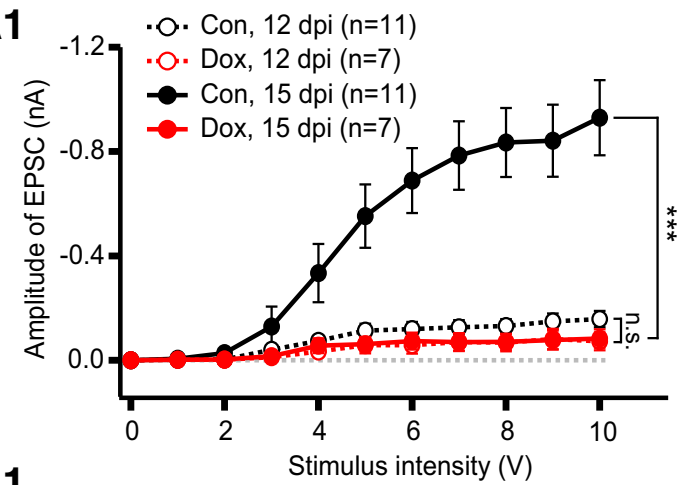

B1

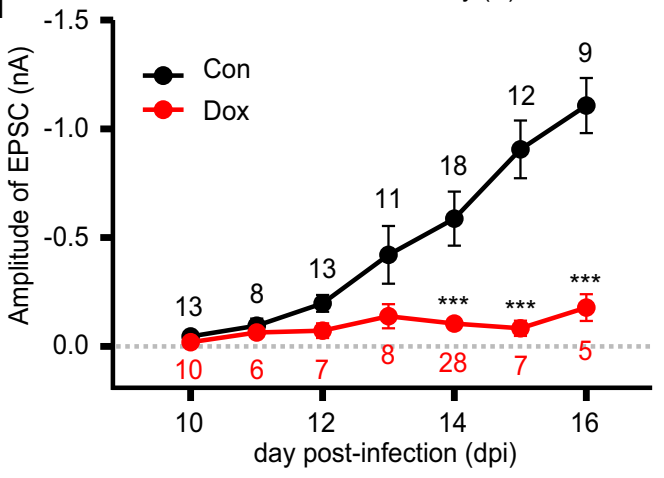

NMDA-EPSC

A2

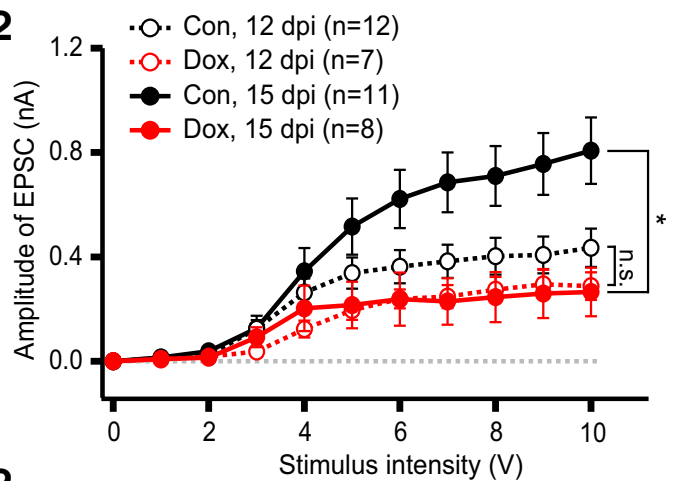

B2

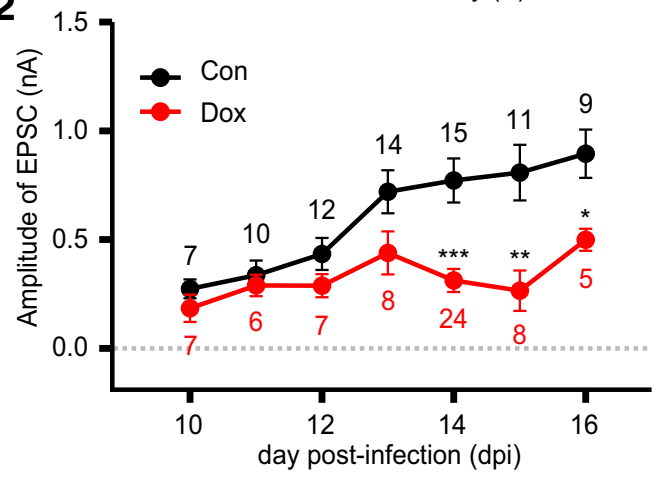

C1

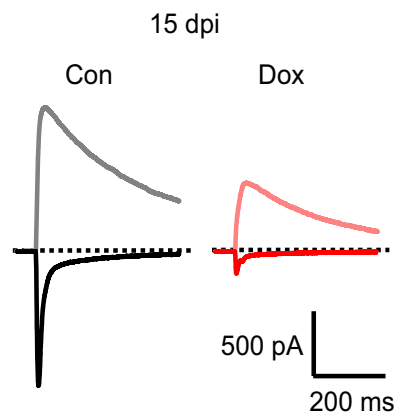

C2

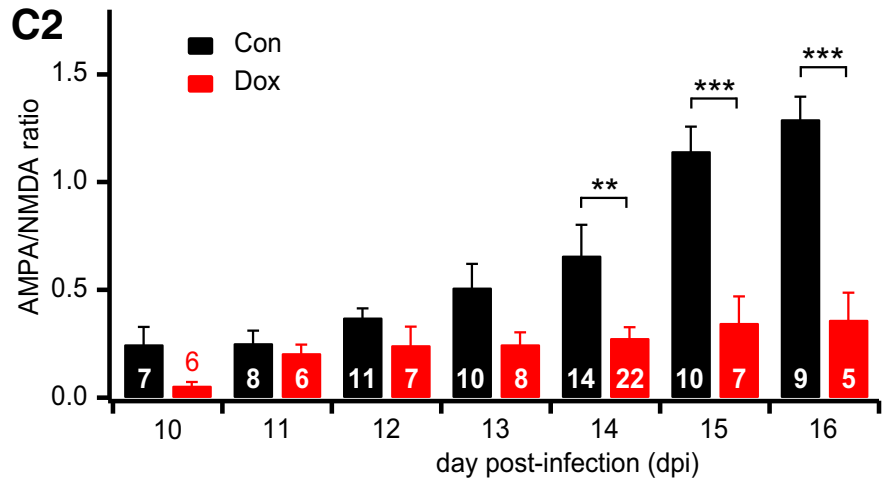

Figure 4. NRG2 is essential for maturation of glutamatergic synapses. A1, A2, Averaged input- output curves of AMPA-EPSCs (A1) and NMDA-EPSCs (A2) in control (black) and NRG2-depleted (red, Dox-treated from $7 \mathrm{dpi}$ ) newborn GCs at 12 dpi (open circles, dotted lines) or at $15 \mathrm{dpi}$ (solid circles, solid lines). Amplitudes of both AMPA-EPSCs and NMDA-EPSCs were significantly lower in Dox-treated GCs at 15 dpi but not at 12 dpi. B, Developmental increases of AMPA-EPSCS (B1) and NMDA-EPSCS (B2) in control and NRG2-depleted (Dox-treated from 7 dpi) newborn GCS. Mean amplitudes of EPSCs evoked by $10 \mathrm{~V}$ stimulation of IML were plotted as a function of dpi. C1, Representative traces of AMPA-EPSCs and NMDA-EPSCs under control (left) and Dox-treated conditions (right) at 15 dpi. C2, Ratio of AMPA-EPSC to NMDA-EPSC amplitude in control and NRG2-depleted (Dox-treated from 7 dpi) newborn GCS.

dpi, NRG2 may have a role in the morphological development rather than glutamatergic synaptogenesis during this period. However, it is not clear whether the lack of developmental increase in the AMPA/NMDA ratio is caused by NRG2 depletion itself or secondary to impaired dendritic arborization (see Discussion).

GABAergic synapse formation of newborn GCs is mediated by ErbB4 tyrosine kinase

Neuregulins are known as ligands of ErbB family of receptor tyrosine kinases (Iwakura and Nawa, 2013). Although NRG2 can bind to both ErbB3 and ErbB4, the NRG2-induced tyrosine kinase activity may be mediated by ErbB4 because ErbB3 lacks kinase activity. To test the involvement of ErbB4 in the downstream signaling of NRG2, we investigated the effect of AG-1478 $(10 \mu \mathrm{M})$, an ErbB4 tyrosine kinase inhibitor, on postsynaptic currents in newborn GCs. The amplitudes of feedforward GPSCs recorded in newborn GCs at 7 dpi was significantly lower in the slice culture treated with AG-1478 from 4 dpi (Fig. 6A1, control vs AG-1478-treated, $p<0.001)$. However, treatment with AG1478 from 10 dpi had little effect on the amplitudes of feedforward GPSCs measured in newborn GCs at 14 dpi (Fig. 6A2, control vs AG-1478-treated, $p=0.878$ ). These results suggest that activation of ErbB4 is required for the developmental increase in GPSCs during the critical period, but not for the later period. Such age dependence of the AG-1478 effect is similar to that of the NRG2 knock-down effects on GPSCs (Fig. 3), suggesting that NRG2 and ErbB4 are on the same signaling pathway required for GABAergic synapse formation.

It is possible that AG-1478 may have an effect on glutamatergic synapses on GABAergic interneurons, which in turn may suppress the feedforward GPSCs in newborn GCs. To rule out this possibility, we recorded the monosynaptic GPSCs in AG-1478treated or control newborn GCs. Consistent with the results of 
A

A $10 \mathrm{dpi}$
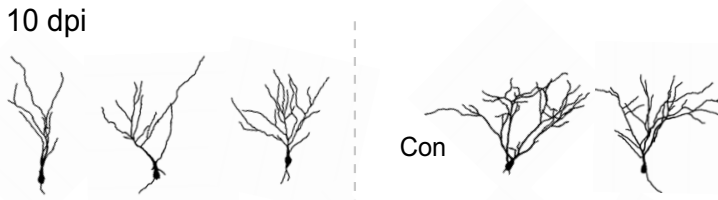

$14 \mathrm{dpi}$

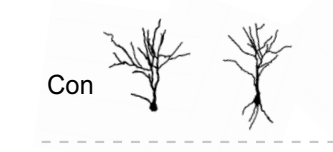

$\underset{\text { Dom Tx. }}{\text { fromi }}$
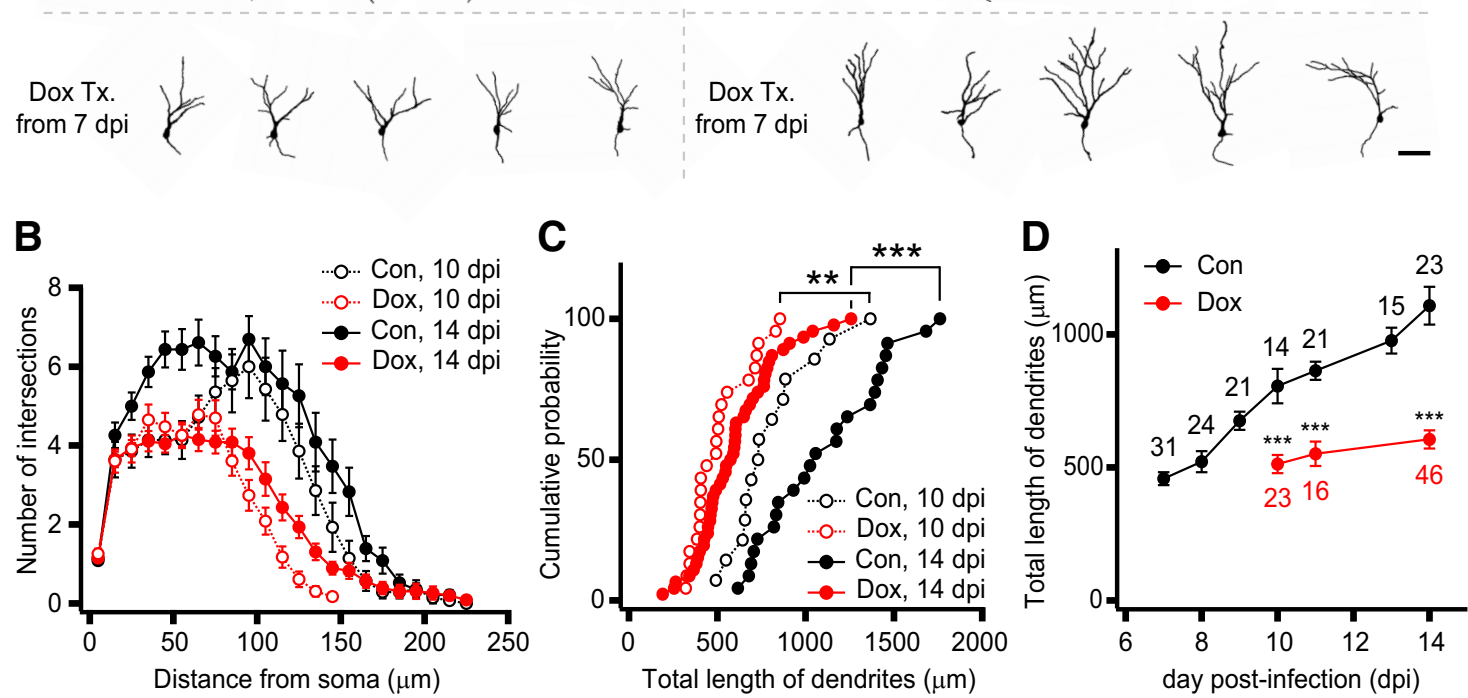

Figure 5. Impairment in dendritic growth precedes that in EPSCs in NRG2-depleted GCS. A, Representative dendritic arbors reconstructed from confocal images of control and Dox-treated newborn GCs at 10 and $14 \mathrm{dpi}$. Scale bar, $50 \mu \mathrm{m}$. B. Sholl analysis of dendritic complexity at 10 and 14 dpi showing the intersection numbers per $10 \mu \mathrm{m}$ radial unit distance. The complexity was significantly reduced by NRG2 depletion at both 10 and 14 dpi (control vs Dox, $F_{(1,35)}=19.1$ at $10 \mathrm{dpi}, F_{(1,67)}=42.5$ at $14 \mathrm{dpi}, p<0.001$ for both dpi's, RM one-way ANOVA). C, Cumulative distributions of dendrites at 10 and 14 dpi. ${ }^{* *} p<0.01 ;{ }^{* * *} p<0,001$ by K-S test. $D$, Developmental increases of total dendritic lengths in control and Dox-treated GCS. ${ }^{* * *} p<0.001$ ( $t$ test).

feedforward GPSCs, the amplitudes of monosynaptic GPSCs were lower in newborn GCs treated with AG-1478 from 4 dpi (Fig. $6 B$, control vs AG-1478-treated, $p<0.01$ ) and were not affected by AG-1478 treatment from 10 dpi (Fig. $6 B, p=0.182$ ), arguing against the possible effects of AG-1478 on glutamatergic synapses onto interneurons. The inhibition effect of AG-1478 was significantly larger than the knock-down effect of NRG2 on feedforward GPSCs (Fig. 6A1, Dox-treated vs AG-1478-treated, $p=0.034$ ). The reason for this might be either that the efficiency of NRG2 knock-down is $<100 \%$ or that the drug effect is immediate whereas the knock-down of preexisting NRG2 takes effect later. To confirm the involvement of ErbB4, we tested the effect of monoclonal antibody targeting ectodomain of ErbB4 (C-erbB4 Ab-3 antibody, denoted as "Ab-3"). The ErbB4-blocking effect of Ab-3 antibody has been tested previously (Min et al., 2011). Treatment of the slice culture with Ab-3 antibody $(10 \mu \mathrm{g} / \mathrm{ml})$ from 4 dpi suppressed monosynaptic GPSCs measured at $14 \mathrm{dpi}$ to a similar extent as AG-1478, confirming the involvement of ErbB4 in GABAergic synaptogenesis. The PPR was not altered by any of NRG2 depletion or AG-1478 or Ab-3 treatments from 4 dpi ( $p>0.05$ for all three conditions), implying that the decrease of monosynaptic GPSC amplitude by NRG2 knock-down or by ErbB4 inhibition is not caused by a change in presynaptic release probability.

Because NRG2 plays an essential role in the development of, not only GABAergic, but also glutamatergic synapses in newborn GCs, we tested whether ErbB4 mediates the downstream signaling of NRG2 involved in the latter. Unlike GPSCs, neither AMPA-EPSCs nor NMDA-EPSCs recorded in newborn GCs at 14 dpi were affected by AG-1478 treatment from $7 \mathrm{dpi}$ (control vs AG-1478-treated; Fig. 6C1, AMPA-EPSCs, $p=0.302$; Fig. $6 D 1$, NMDA-EPSCs, $p=0.942$ ). The PPRs of AMPA-EPSCs and NMDA-EPSCs were not altered by either Dox- or AG-1478 treat- ment from $7 \mathrm{dpi}$ (Fig. $6 C 2, D 2$ ). These results suggest that the role of NRG2 in glutamatergic synapse development in newborn GC is independent of ErbB4 tyrosine kinase.

\section{NRG2 signaling for glutamatergic synapse maturation and dendritic growth is mediated by its intracellular domain} Given that ErbB4 kinase activity is not relevant to the NRG2dependent development of glutamatergic synapses in newborn GCs, we hypothesized that a reverse signaling pathway of NRG2 is involved. The role of reverse signaling of NRG1 has been studied at glutamatergic synapses of central neurons for the last decade (Bao et al., 2003; Bao et al., 2004; Chen et al., 2010; Fazzari et al., 2014) and involves activity-dependent cleavage and nuclear translocation of intracellular domain of NRG1 (NRG1-ICD). To investigate the possible involvement of NRG2-ICD in glutamatergic synapse development, we designed a retroviral vector expressing myc-tagged NRG2-ICD and TagRFP under the control of Tet-On and LTR promoters, respectively (Fig. 7A1). Newborn GCs infected by retrovirus encoding NRG2-ICD could be distinguished from those infected by retrovirus encoding shNRG2mir by the red fluorescence of TagRFP. Using immunohistochemical detection of myc, we confirmed that the newborn GCs infected by the retrovirus reliably express myc-tagged NRG2-ICD in the presence of Dox (Fig. 7A2).

Next, we tested whether the NRG2 knock-down effects on glutamatergic synaptic transmission and dendritic arborization could be rescued by the expression of NRG2-ICD. Hippocampal slice cultures were simultaneously infected with two kinds of retroviruses: retroviruses encoding shNRG2mir plus EGFP (shNRG2mir virus) or NRG2-ICD plus TagRFP (NRG2-ICD virus). Because shNRG2mir targets a different domain of NRG2 other than its ICD, it is expected that endogenous NRG2 is depleted by shNRG2mir, whereas shNRG2mir-resistant NRG2- 


\section{Feed-forward GPSC}

A1

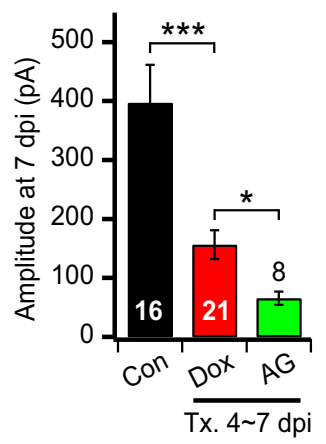

A2

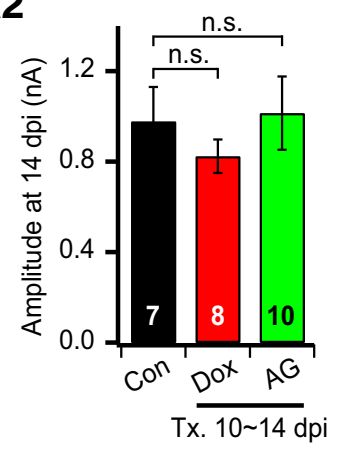

Monosynaptic GPSC

B

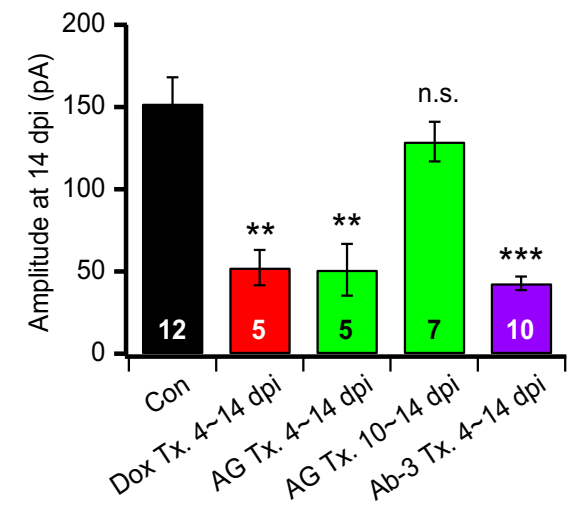

NMDA-EPSC

AMPA-EPSC
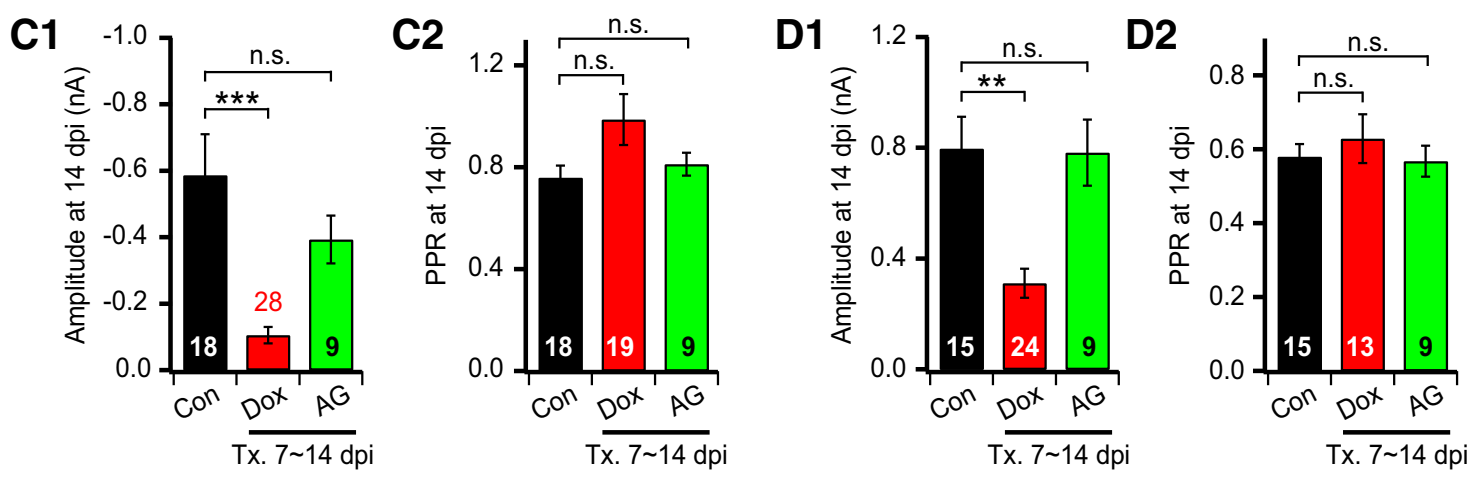

Figure 6. Effects of ErbB4 inhibition on GPSCs and EPSCS. $\boldsymbol{A}, \boldsymbol{B}$, Effects of ErbB4 inhibitors on GPSCs of newborn GCS. $\boldsymbol{A} 1, \boldsymbol{A 2}$, Mean amplitudes of feedforward GPSCs in control (black), Dox-treated (red), and AG-1478-treated (10 $\mu \mathrm{m}$, green, denoted as "AG") newborn GCs. Dox and AG-1478 were added in culture media from $4 \mathrm{dpi}(\boldsymbol{A 1})$ or $10 \mathrm{dpi}(\boldsymbol{A 2})$ and feedforward GPSCS were recorded at $7 \mathrm{dpi}(\boldsymbol{A} 1)$ or $14 \mathrm{dpi}(\boldsymbol{A 2})$, respectively. B, Mean amplitudes of monosynaptic GPSCs at 14 dpi in newborn GCs grown under control conditions or under the condition in the presence of Dox, AG-1478, or anti-ErbB4 antibody ( $10 \mu \mathrm{g} / \mathrm{ml}$, purple, denoted as "Ab-3"). Note that the three kinds of treatment reduced the GPSCs to a similar extent when the starting date of treatment was $4 \mathrm{dpi}$. C, D, Effects of AG-1478 on EPSCs in newborn GCS. Mean amplitudes (C1, D1) and PPR (C2, D2) of AMPA-EPSCS (C) and those of NMDA-EPSCS (D) in control, Dox-treated, or AG-1478-treated newborn GCS. Dox and AG-1478 were added in culture media from $7 \mathrm{dpi}$ and EPSCs were recorded at $14 \mathrm{dpi}$. Tx., Treatment.

ICD is overexpressed in the newborn GCs labeled with both EGFP and TagRFP (Fig. 7A3). We compared postsynaptic currents in newborn GCs under four different conditions as defined in Figure $7 A 4$. Dox treatment started at 7 and 4 dpi to observe EPSCs and GPSCs, respectively. We found that neither the amplitudes of AMPA-EPSCs and NMDA-EPSCs nor the AMPA/ NMDA ratio was significantly different in the doubly labeled newborn GCs (denoted as "rescue" in Fig. 7) compared with the control GCs, indicating that the effects of NRG2 depletion is rescued to the control level by the NRG2-ICD overexpression (Figs. $7 B 1-B 3$, control vs rescue, $p>0.1$ ). Conversely, the impaired development of GPSCs caused by NRG2 knock-down was not rescued by NRG2-ICD overexpression (Fig. 7C, NRG2 K/D vs rescue, $p=0.697)$. These results demonstrate that reverse signaling of NRG2, mediated by NRG2-ICD, is involved in the development of glutamatergic synapses, but not in GABAergic synapse formation. Finally, we observed the dendritic morphology of EGFP-positive and TagRFP-positive newborn GCs treated with Dox from $7 \mathrm{dpi}$. Overexpression of NRG2-ICD rescued the impairments of dendritic arborization observed in NRG2depleted newborn GCs at 14 dpi (Fig. 7D1). Both the complexity of dendritic arbor and the total dendritic length were rescued to the control level by NRG2-ICD overexpression (Fig. 7D2, Scholl analysis, $F_{(1,31)}=0.074, p=0.788$, RM one-way ANOVA; Fig. $7 D 3$, cumulative distributions of total dendritic lengths, $p=$ 0.991, K-S test; Fig. 7D4, mean total dendritic length, $p<0.001$, $t$ test). These results suggest that reverse signaling of NRG2 is involved in the maturation of glutamatergic synapses and dendritic growth in newborn GCs, whereas forward signaling of NRG2 contributes to GABAergic synaptogenesis.

\section{Discussion}

The present study indicates that NRG2 plays a key role in formation of GABAergic synapses and maturation of glutamatergic synapses in newborn GCs via distinct signaling pathways: forward and reverse signaling of NRG2, respectively. NRG2 expression is very low at birth and notably increases during PW1 in neocortex, hippocampus, and cerebellum with development and decreases by PW3 in most brain regions except dentate gyrus, olfactory bulb, and cerebellum (Longart et al., 2004). Given that GABAergic synaptogenesis starts during PW2 in newborn GCs (Fig. $2 F$ ), it may coincide with the period when NRG2 expression is fully matured.

Because the expression of NRG2 is confined to somatodendritic compartments (Longart et al., 2004), NRG2 can act as a ligand for presynaptic ErbB4. Recent immunochemical studies revealed that ErbB4 is expressed on parvalbumin (PV) or cholecystokinin-positive basket cells and neuronal nitric oxide synthase (nNOS)-positive interneurons (Neddens and Buonanno, 2010). Neurogliaform interneurons are a major group of nNOS-positive interneurons (Armstrong et al., 2012). Interestingly, it has been shown recently that newborn 


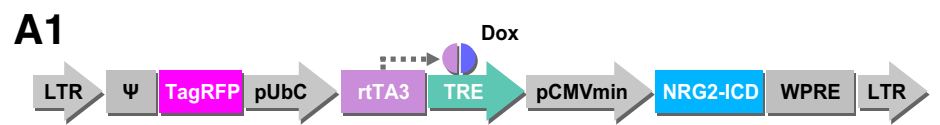

\begin{tabular}{|c|c|c|c|c|c|c|}
\hline virus type & \multicolumn{2}{|c|}{ shNRG2mir virus } & \multicolumn{2}{|c|}{ NRG2-ICD virus } & \multicolumn{2}{|c|}{ Both } \\
\hline Dox Tx. & - & + & - & + & - & + \\
\hline notation & Control & $\mathrm{NRG} 2 \mathrm{~K} / \mathrm{D}$ & & ICD O/E & & Rescue \\
\hline
\end{tabular}

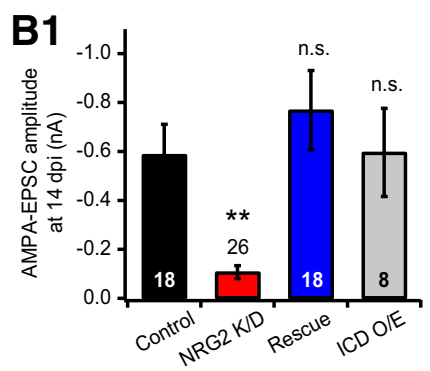

D1 $14 \mathrm{dpi}$

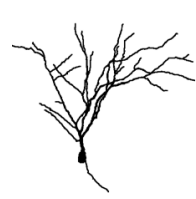

Control
B2

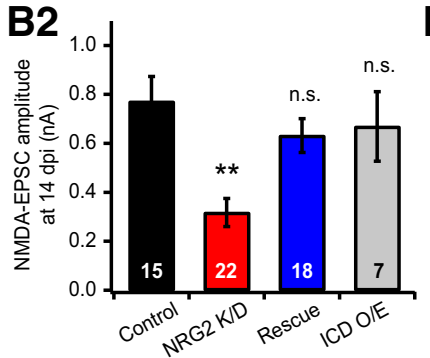

\section{D2}

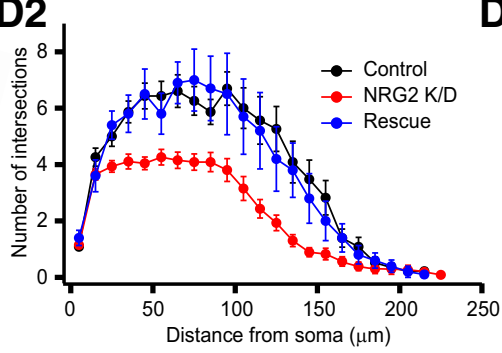

B3
A2
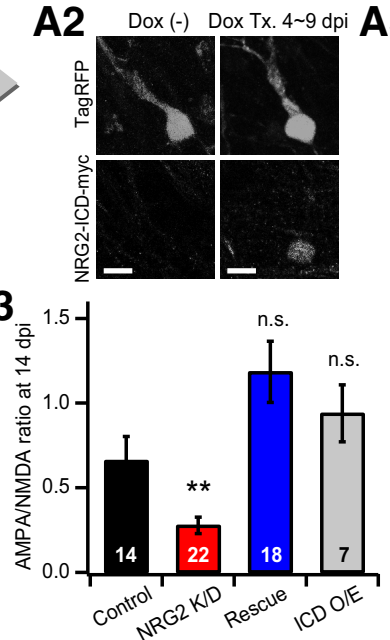

D3

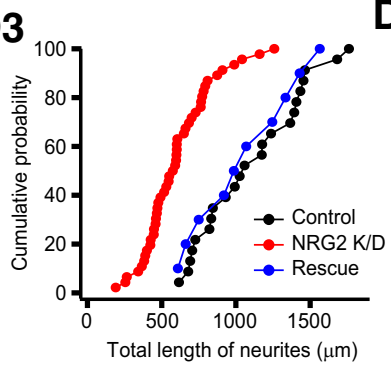

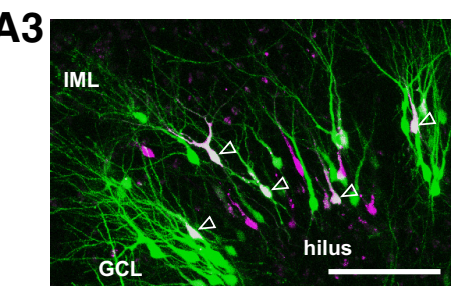

C $1 . 2 \longdiv { * * }$

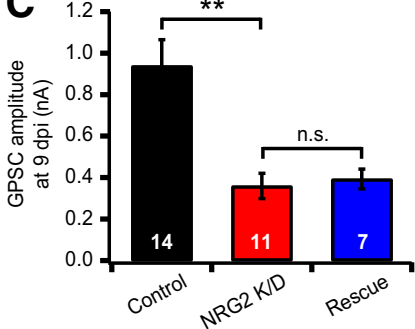

D4

Figure 7. Effects of NRG2 depletion on glutamatergic synaptic transmission and dendritic arborization are rescued by NRG2-ICD overexpression. $A$, Retrovirus for overexpression of intracellular domain of NRG2 (NRG2-ICD) in newborn GCS. A1, Schematic of the retroviral cassette for tetracycline-inducible expression of NRG2-ICD in parallel with constitutive expression of TagRFP. A2, Only under the Dox-treated conditions (Dox TX.) did the TagRFP-positive newborn GCs express c-myc-tagged NRG2-ICD. NRG2-ICD was immunostained with anti-myc lgG. Scale bar, $10 \mu \mathrm{m}$. A3, Fluorescence image of a hippocampal slice culture infected with two kinds of retroviruses: shNRG2mir virus and NRG2-ICD virus. Newborn GCs infected with one of the two viruses express EGFP (green) or TagRFP (magenta). Open arrowheads indicate the newborn GCs infected with both viruses simultaneously. Scale bar, $100 \mu \mathrm{m}$. $A 4$, Notations for experimental conditions of virus types and Dox treatment. B, C, Concurrent overexpression of NRG2-ICD in the NRG2-depeleted newborn GCs rescued the knock-down effects of shNRG2mir on AMPA-EPSCS (B1), NMDA-EPSCs (B2), and the AMPA/NMDA ratio (B3), but not GPSCS (C). The amplitudes of AMPA-EPSCs or NMDA-EPSCs of newborn GCs overexpressing only NRG2-ICD were not significantly different (B1-B3, gray bars) from the control GCs. Dox was applied from 7 to $14 \mathrm{dpi}$ (B1-B3) or from 4 to $9 \mathrm{dpi}$ (C).D, Overexpression of NRG2-ICD rescued the impaired dendritic arborization caused by NRG2 knock-down (control, $n=13 ; \mathrm{NRG} \mathrm{K/D,} n=27$; rescue, $n=10)$. D1, Representative morphology of control, NRG2 K/D, and rescued newborn G(s at $14 \mathrm{dpi}$. Scale bar, $50 \mu \mathrm{m}$. D2, Dendritic arbor complexity evaluated by Sholl analysis was not different between control and rescued GCs (control vs rescued, $F_{(1,21)}=2.45, p=0.774$ by RM one-way ANOVA). D3, D4, Cumulative distribution (D3) and averages (D4) of total length of dendrites were rescued by NRG2-ICD to the control level (control vs rescue; D3, $p=0.943, \mathrm{~K}$-S test; $\mathbf{D} 4, p=0.476$ ). Results for control and NRG2-depleted newborn GCS were reproduced from those in Figure $5, B-D$.

GCs receive synaptic inputs from PV-positive and neurogliaform interneurons (Markwardt et al., 2011; Song et al., 2013). It has been suggested that presynaptic terminals of GABAergic interneurons innervating perisomatic regions of principal neurons express ErbB4 and that NRG1-dependent activation of ErbB4 promotes GABAergic synaptogenesis in cortical neurons (Woo et al., 2007; Fazzari et al., 2010; but see Vullhorst et al., 2009). Consistent with these notions, we found that GABAergic synaptogenesis in newborn GCs is suppressed, not only by NRG2 depletion, but also by inhibition of ErbB4. These findings raise the possibility that ErbB4 localized at GABAergic presynaptic terminals, probably of basket cells and/or neurogliaform interneurons, may be responsible for the NRG2-dependent GABAergic synaptogenesis in newborn GCs. Because the expression of ErbB4 in newborn GCs is very weak, it is unlikely that postsynaptic NRG2 on newborn GCs act as an autocrine ligand for postsynaptic ErbB4 even if its ectodomain is cleaved.

In contrast to our finding that NRG2 is dispensable for the maintenance of GABAergic synapses in newborn GCs, recent studies showed that the NRG1/ErbB4 interaction enhances GABAergic transmission in neocortex and amygdala (Woo et al., 2007; Lu et al., 2014). This discrepancy may be attributed to the molecular difference between NRG1 and NRG2, to the differences in developmental stages, and/or to the regional difference in the basal interaction level between ErbB4 and neuregulins.

There are controversial studies on the role of ErbB4 in glutamatergic synapse maturation. The lack of AG-1478 effects on the development of glutamatergic synapses suggests that the tyrosine kinase activity of ErbB4 is not involved in glutamatergic synapse maturation (Fig. 6C,D). It has been suggested, however, that presynaptic NRG1-induced activation of postsynaptic ErbB4 enhances AMPA-EPSCs in a kinase-dependent manner (Li et al., 2007) and that ErbB2/4 is involved in the maturation of dendritic spines (Barros et al., 2009). This idea on the role of ErbB4 in glutamatergic synapse maturation was challenged by a more recent study showing little role of ErbB4 expressed on pyramidal neurons in excitatory synaptic transmission (Fazzari et al., 2010). Therefore, the present study is rather consistent with the latter view. Nevertheless, our results do not rule out the involvement of ErbB4 in glutamatergic synapse maturation because interaction of NRG2 with the ectodomain of ErbB4 may trigger the reverse signaling of NRG2 independently of the ErbB4 kinase activity (Bao et al., 2003; Chen et al., 2010). 


\section{Reverse signaling of neuregulins}

We showed that the NRG2 knock-down effects on the glutamatergic synapse maturation and dendritic arborization are rescued by the expression of NRG2-ICD (Fig. 7). Furthermore, the development of glutamatergic synapses was independent of ErbB4 tyrosine kinase activity (Fig. 6C,D). These results suggest that maturation of glutamatergic synapse is mediated, not by forward signaling, but rather by reverse signaling of NRG2. Although reverse signaling of NRG2 has not been studied previously, there is a line of evidence for the reverse signaling of NRG1 type III (Bao et al., 2003; Bao et al., 2004; Chen et al., 2010; Fazzari et al., 2014). The reverse signaling of NRG1 type III requires sequential cleavage of ectodomain and transmembrane domain of NRG1 by TNF- $\alpha$ converting enzyme (TACE) and $\gamma$-secretase containing the subunit Aphlb, respectively (Bao et al., 2003; La Marca et al., 2011; Fazzari et al., 2014). The cleaved NRG1ICDs are translocated into the nucleus and enhance transcription of PSD-95 by interaction with a zinc-finger transcription factor, Eos (Bao et al., 2004). Therefore, any genetic mutations in $\gamma$-secretasedependent intramembranous cleavage site of NRG1 or Aph1b resulted in impairments of dendritic arborization, dendritic spine formation, and glutamatergic synaptic transmission (Chen et al., 2010; Fazzari et al., 2014).

Examining the amino acid sequences of NRG2, we found that the $\gamma$-secretase and TACE-dependent cleavage sites, as well as the nuclear localization signal, are conserved in NRG2. Because the key sequences are not different between the $\alpha$ and $\beta$ isoforms of NRG2, both isoforms may mediate reverse signaling (Hobbs et al., 2002). In light of knowledge from NRG1 studies, there is a possibility that NRG2-ICD may function as a transcription regulator, but the identity of transcription factors interacting with NRG2-ICD remains to be elucidated.

The expression and cleavage of NRG1 are regulated in an activity-dependent manner (Eilam et al., 1998; Bao et al., 2003; Bao et al., 2004; Ozaki et al., 2004), implying that GABAergic input-induced $\mathrm{Ca}^{2+}$ signaling may be one of factors prerequisite for the reverse signaling of NRG2 in newborn GCs. Previous studies suggested that excitatory GABAergic inputs and subsequent $\mathrm{Ca}^{2+}$ signaling play a key role in coupling the neural activity and the structural plasticity of newborn GCs (Tozuka et al., 2005; Ge et al., 2006; Lee et al., 2012a). Therefore, it is possible that the nurturing effects of network activity on dendritic arborization in newborn GCs may require the reverse signaling of NRG2 as a downstream signaling pathway of the depolarizing GABAergic inputs.

\section{Role of NRG2 in dendritic growth and maturation of glutamatergic synapses}

Given that only NMDA receptors are expressed in nascent glutamatergic synapses, the amplitude of NMDA-EPSCs could be a more appropriate parameter for glutamatergic synaptogenesis than AMAP-EPSCs. The suppression of developmental increase in NMDA-EPSCs can be caused by direct inhibition of glutamatergic synaptogenesis or secondary to the inhibition of dendritic arborization. The present study showed that depletion of NRG2 impairs dendritic development, with little effect on NMDAEPSCs between 10 and 13 dpi (Figs. 4B2, 5). Therefore, it is unlikely that NRG2 contributes to glutamatergic synaptogenesis. In addition, the impoverished dendritic arbor caused by NRG2 depletion may be responsible for the lower developmental increase of EPSCs that became evident at 14 dpi in Dox-treated GCs. It remains to be elucidated, however, whether maturation of glutamatergic synapses, manifested as an increase in the AMPA/ NMDA ratio, is mediated directly by NRG2 or if it is secondary to dendritic growth. Because the effects of NRG2 depletion on the NMDA-EPSCs emerged at the dpi, when AMPA-EPSCs started to increase and its effect on AMPA-EPSCs was more substantial, it is likely that NRG2 contributes directly to maturation of glutamatergic synapses. Differential evaluation of dendritic growth and maturation of glutamatergic synapses in immature GCs may not be easy, especially if dendritic arborization in these cells follows the synaptotrophic hypothesis, which argues that addition of AMPA receptors to a nascent glutamatergic synapse is required for stabilization, not only of the synapse, but also of the associated dendritic branch (Haas et al., 2006; Cline and Haas, 2008). Investigation of NRG2 knock-down effects on EPSCs in mature GCs may help to address this question.

\section{Maturation of newborn GCs in hippocampal slice cultures}

In the present study, we investigated the role of NRG2 by comparing developmental increases in synaptic currents in newborn GCs labeled in organotypic slice cultures taken from the early postnatal rat hippocampus. Two factors should be considered for evaluating the ex vivo data. First, maturation of newborn GCs is known to be faster at a younger age (Overstreet-Wadiche et al., 2006a). As noted in the present study and our previous study (Lee et al., 2012a), GABAergic synapse formation in newborn GCs in the present study was 3 d earlier than that of newborn GCs in the adult hippocampus (Espósito et al., 2005). Second, the development of newborn GCs is affected by abnormal patterns of neural activity of the slice culture. It has been shown that rewiring subsequent to slicing-induced massive deafferentiation leads to epileptiform discharge in the CA3 region (Dyhrfjeld-Johnsen et al., 2010). Given that seizure accelerates the maturation of newborn GCs (Overstreet-Wadiche et al., 2006b), the time course of developmental increases of synaptic current and morphological maturation may be faster than in vivo. Nevertheless, maturation of newborn GCs in the organotypic slice culture was comparable to that in age-matched in vivo conditions (Namba et al., 2007). Indeed, we found that the amplitude of monosynaptic GPSCs reached a plateau 1 or $2 \mathrm{~d}$ earlier in the slice culture than newborn GCs labeled in vivo of P16-P22 rats (data not shown). This small difference may be related to the delayed emergence of epileptiform discharge in the culture. It has been shown that epileptiform discharges were first observed at $21 \mathrm{dpi}$ in $50 \%$ of the cultures, indicating that our observation window is earlier than the development of epileptiform discharge.

The present study was done in the neonatal or juvenile dentate gyrus, but adult neurogenesis recapitulates embryonic development (Espósito et al., 2005). Furthermore, the expression of NRG2 persists in the mature GCs. Therefore, it remains to be elucidated whether NRG2 may play a role in synaptogenesis and/or synaptic plasticity in mature GCs and adult-born GCs.

\section{References}

Abe Y, Namba H, Kato T, Iwakura Y, Nawa H (2011) Neuregulin-1 signals from the periphery regulate AMPA receptor sensitivity and expression in GABAergic interneurons in developing neocortex. J Neurosci 31: 5699-5709. CrossRef Medline

Akerman CJ, Cline HT (2006) Depolarizing GABAergic conductances regulate the balance of excitation to inhibition in the developing retinotectal circuit in vivo. J Neurosci 26:5117-5130. CrossRef Medline

Anton ES, Ghashghaei HT, Weber JL, McCann C, Fischer TM, Cheung ID, Gassmann M, Messing A, Klein R, Schwab MH, Lloyd KC, Lai C (2004) Receptor tyrosine kinase ErbB4 modulates neuroblast migration and placement in the adult forebrain. Nat Neurosci 7:1319-1328. CrossRef Medline

Armstrong C, Krook-Magnuson E, Soltesz I (2012) Neurogliaform and Ivy 
cells: a major family of nNOS expressing GABAergic neurons. Front Neural Circuits 6:23. Medline

Bao J, Wolpowitz D, Role LW, Talmage DA (2003) Back signaling by the Nrg-1 intracellular domain. J Cell Biol 161:1133-1141. CrossRef Medline

Bao J, Lin H, Ouyang Y, Lei D, Osman A, Kim TW, Mei L, Dai P, Ohlemiller KK, Ambron RT (2004) Activity-dependent transcription regulation of PSD-95 by neuregulin-1 and Eos. Nat Neurosci 7:1250-1258. CrossRef Medline

Barros CS, Calabrese B, Chamero P, Roberts AJ, Korzus E, Lloyd K, Stowers L, Mayford M, Halpain S, Müller U (2009) Impaired maturation of dendritic spines without disorganization of cortical cell layers in mice lacking NRG1/ErbB signaling in the central nervous system. Proc Natl Acad Sci U S A 106:4507-4512. CrossRef Medline

Bartos M, Vida I, Frotscher M, Geiger JR, Jonas P (2001) Rapid signaling at inhibitory synapses in a dentate gyrus interneuron network. J Neurosci 21:2687-2698. Medline

Buckmaster PS, Strowbridge BW, Kunkel DD, Schmiege DL, Schwartzkroin PA (1992) Mossy cell axonal projections to the dentate gyrus molecular layer in the rat hippocampal slice. Hippocampus 2:349-362. CrossRef Medline

Busfield SJ, Michnick DA, Chickering TW, Revett TL, Ma J, Woolf EA, Comrack CA, Dussault BJ, Woolf J, Goodearl AD, Gearing DP (1997) Characterization of a neuregulin-related gene, Don-1, that is highly expressed in restricted regions of the cerebellum and hippocampus. Mol Cell Biol 17:4007-4014. CrossRef Medline

Carraway KL 3rd, Weber JL, Unger MJ, Ledesma J, Yu N, Gassmann M, Lai C (1997) Neuregulin-2, a new ligand of ErbB3/ErbB4-receptor tyrosine kinases. Nature 387:512-516. CrossRef Medline

Chancey JH, Poulsen DJ, Wadiche JI, Overstreet-Wadiche L (2014) Hilar mossy cells provide the first glutamatergic synapses to adult-born dentate granule cells. J Neurosci 34:2349-2354. CrossRef Medline

Chang H, Riese DJ 2nd, Gilbert W, Stern DF, McMahan UJ (1997) Ligands for ErbB-family receptors encoded by a neuregulin-like gene. Nature 387: 509-512. CrossRef Medline

Chen YJ, Johnson MA, Lieberman MD, Goodchild RE, Schobel S, Lewandowski N, Rosoklija G, Liu RC, Gingrich JA, Small S, Moore H, Dwork AJ, Talmage DA, Role LW (2008) Type III neuregulin-1 is required for normal sensorimotor gating, memory-related behaviors, and corticostriatal circuit components. J Neurosci 28:6872-6883. CrossRef Medline

Chen Y, Hancock ML, Role LW, Talmage DA (2010) Intramembranous valine linked to schizophrenia is required for neuregulin 1 regulation of the morphological development of cortical neurons. J Neurosci 30: 9199-9208. CrossRef Medline

Cline H, Haas K (2008) The regulation of dendritic arbor development and plasticity by glutamatergic synaptic input: a review of the synaptotrophic hypothesis. J Physiol 586:1509-1517. CrossRef Medline

Corfas G, Rosen KM, Aratake H, Krauss R, Fischbach GD (1995) Differential expression of ARIA isoforms in the rat brain. Neuron 14:103-115. CrossRef Medline

Dickins RA, Hemann MT, Zilfou JT, Simpson DR, Ibarra I, Hannon GJ, Lowe SW (2005) Probing tumor phenotypes using stable and regulated synthetic microRNA precursors. Nat Genet 37:1289-1295. Medline

Dvorzhak A, Semtner M, Faber DS, Grantyn R (2013) Tonic mGluR5/CB1dependent suppression of inhibition as a pathophysiological hallmark in the striatum of mice carrying a mutant form of huntingtin. J Physiol 591:1145-1166. CrossRef Medline

Dyhrfjeld-Johnsen J, Berdichevsky Y, Swiercz W, Sabolek H, Staley KJ (2010) Interictal spikes precede ictal discharges in an organotypic hippocampal slice culture model of epileptogenesis. J Clin Neurophysiol 27:418-424. CrossRef Medline

Eilam R, Pinkas-Kramarski R, Ratzkin BJ, Segal M, Yarden Y (1998) Activity-dependent regulation of Neu differentiation factor/neuregulin expression in rat brain. Proc Natl Acad Sci U S A 95:1888-1893. CrossRef Medline

Espósito MS, Piatti VC, Laplagne DA, Morgenstern NA, Ferrari CC, Pitossi FJ, Schinder AF (2005) Neuronal differentiation in the adult hippocampus recapitulates embryonic development. J Neurosci 25:10074-10086. CrossRef Medline

Fazzari P, Paternain AV, Valiente M, Pla R, Luján R, Lloyd K, Lerma J, Marín O, Rico B (2010) Control of cortical GABA circuitry development by Nrg1 and ErbB4 signalling. Nature 464:1376-1380. CrossRef Medline
Fazzari P, Snellinx A, Sabanov V, Ahmed T, Serneels L, Gartner A, Shariati SA, Balschun D, De Strooper B (2014) Cell autonomous regulation of hippocampal circuitry via Aphlb-gamma-secretase/neuregulin 1 signalling. Elife 2.

Ge S, Goh EL, Sailor KA, Kitabatake Y, Ming GL, Song H (2006) GABA regulates synaptic integration of newly generated neurons in the adult brain. Nature 439:589-593. CrossRef Medline

Ghashghaei HT, Weber J, Pevny L, Schmid R, Schwab MH, Lloyd KC, Eisenstat DD, Lai C, Anton ES (2006) The role of neuregulin-ErbB4 interactions on the proliferation and organization of cells in the subventricular zone. Proc Natl Acad Sci U S A 103:1930-1935. CrossRef Medline

Gossen M, Bujard H (1992) Tight control of gene expression in mammalian cells by tetracycline-responsive promoters. Proc Natl Acad Sci U S A 89: 5547-5551. CrossRef Medline

Haas K, Li J, Cline HT (2006) AMPA receptors regulate experiencedependent dendritic arbor growth in vivo. Proc Natl Acad Sci U S A 103: 12127-12131. CrossRef Medline

Hobbs SS, Coffing SL, Le AT, Cameron EM, Williams EE, Andrew M, Blommel EN, Hammer RP, Chang H, Riese DJ 2nd (2002) Neuregulin isoforms exhibit distinct patterns of ErbB family receptor activation. Oncogene 21:8442-8452. CrossRef Medline

Hollrigel GS, Ross ST, Soltesz I (1998) Temporal patterns and depolarizing actions of spontaneous GABAA receptor activation in granule cells of the early postnatal dentate gyrus. J Neurophysiol 80:2340-2351. Medline

Iwakura Y, Nawa H (2013) ErbB1-4-dependent EGF/neuregulin signals and their cross talk in the central nervous system: pathological implications in schizophrenia and Parkinson's disease. Front Cell Neurosci 7.

La Marca R, Cerri F, Horiuchi K, Bachi A, Feltri ML, Wrabetz L, Blobel CP, Quattrini A, Salzer JL, Taveggia C (2011) TACE (ADAM17) inhibits Schwann cell myelination. Nat Neurosci 14:857-865. CrossRef Medline

Lee D, Lee KH, Ho WK, Lee SH (2007) Target cell-specific involvement of presynaptic mitochondria in post-tetanic potentiation at hippocampal mossy fiber synapses. J Neurosci 27:13603-13613. CrossRef Medline

Lee H, Lee D, Park CH, Ho WK, Lee SH (2012a) GABA mediates the network activity-dependent facilitation of axonal outgrowth from the newborn granule cells in the early postnatal rat hippocampus. Eur J Neurosci 36:2743-2752. CrossRef Medline

Lee KH, Lee JS, Lee D, Seog DH, Lytton J, Ho WK, Lee SH (2012b) KIF21Amediated axonal transport and selective endocytosis underlie the polarized targeting of NCKX2. J Neurosci 32:4102-4117. CrossRef Medline

Li B, Woo RS, Mei L, Malinow R (2007) The neuregulin-1 receptor erbB4 controls glutamatergic synapse maturation and plasticity. Neuron 54: 583-597. CrossRef Medline

Liu X, Bates R, Yin DM, Shen C, Wang F, Su N, Kirov SA, Luo Y, Wang JZ, Xiong WC, Mei L (2011) Specific regulation of NRG1 isoform expression by neuronal activity. J Neurosci 31:8491-8501. CrossRef Medline

Longair MH, Baker DA, Armstrong JD (2011) Simple Neurite Tracer: open source software for reconstruction, visualization and analysis of neuronal processes. Bioinformatics 27:2453-2454. CrossRef Medline

Longart M, Liu Y, Karavanova I, Buonanno A (2004) Neuregulin-2 is developmentally regulated and targeted to dendrites of central neurons. J Comp Neurol 472:156-172. CrossRef Medline

Lu Y, Sun XD, Hou FQ, Bi LL, Yin DM, Liu F, Chen YJ, Bean JC, Jiao HF, Liu X, Li BM, Xiong WC, Gao TM, Mei L (2014) Maintenance of GABAergic activity by neuregulin 1-ErbB4 in amygdala for fear memory. Neuron 84:835-846. CrossRef Medline

Marchionni I, Maccaferri G (2009) Quantitative dynamics and spatial profile of perisomatic GABAergic input during epileptiform synchronization in the CA1 hippocampus. J Physiol 587:5691-5708. CrossRef Medline

Markwardt SJ, Dieni CV, Wadiche JI, Overstreet-Wadiche L (2011) Ivy/ neurogliaform interneurons coordinate activity in the neurogenic niche. Nat Neurosci 14:1407-1409. CrossRef Medline

Mierau SB, Meredith RM, Upton AL, Paulsen O (2004) Dissociation of experience-dependent and -independent changes in excitatory synaptic transmission during development of barrel cortex. Proc Natl Acad Sci U S A 101:15518-15523. CrossRef Medline

Min SS, An J, Lee JH, Seol GH, Im JH, Kim HS, Baik TK, Woo RS (2011) Neuregulin-1 prevents amyloid beta-induced impairment of long-term potentiation in hippocampal slices via ErbB4. Neurosci Lett 505:6-9. CrossRef Medline

Namba T, Mochizuki H, Onodera M, Namiki H, Seki T (2007) Postnatal neurogenesis in hippocampal slice cultures: early in vitro labeling of neu- 
ral precursor cells leads to efficient neuronal production. J Neurosci Res 85:1704-1712. CrossRef Medline

Neddens J, Buonanno A (2010) Selective populations of hippocampal interneurons express ErbB4 and their number and distribution is altered in ErbB4 knockout mice. Hippocampus 20:724-744. Medline

Overstreet-Wadiche LS, Bensen AL, Westbrook GL (2006a) Delayed development of adult-generated granule cells in dentate gyrus. J Neurosci 26: 2326-2334. CrossRef Medline

Overstreet-Wadiche LS, Bromberg DA, Bensen AL, Westbrook GL (2006b) Seizures accelerate functional integration of adult-generated granule cells. J Neurosci 26:4095-4103. CrossRef Medline

Ozaki M, Itoh K, Miyakawa Y, Kishida H, Hashikawa T (2004) Protein processing and releases of neuregulin-1 are regulated in an activitydependent manner. J Neurochem 91:176-188. CrossRef Medline

Rao MS, Shetty AK (2004) Efficacy of doublecortin as a marker to analyse the absolute number anddendritic growth of newly generated neurons in the adult dentate gyrus. Eur J Neurosci 19:234-246. CrossRef Medline

Schwartz N, Schohl A, Ruthazer ES (2011) Activity-dependent transcription of BDNF enhances visual acuity during development. Neuron 70: 455-467. CrossRef Medline

Song J, Sun J, Moss J, Wen Z, Sun GJ, Hsu D, Zhong C, Davoudi H, Christian KM, Toni N, Ming GL, Song H (2013) Parvalbumin interneurons mediate neuronal circuitry-neurogenesis coupling in the adult hippocampus. Nat Neurosci 16:1728-1730. CrossRef Medline

Soriano E, Frotscher M (1989) A GABAergic axo-axonic cell in the fascia dentata controls the main excitatory hippocampal pathway. Brain Res 503:170-174. CrossRef Medline
Ting AK, Chen Y, Wen L, Yin DM, Shen C, Tao Y, Liu X, Xiong WC, Mei L (2011) Neuregulin 1 promotes excitatory synapse development and function in GABAergic interneurons. J Neurosci 31:15-25. CrossRef Medline

Tozuka Y, Fukuda S, Namba T, Seki T, Hisatsune T (2005) GABAergic excitation promotes neuronal differentiation in adult hippocampal progenitor cells. Neuron 47:803-815. CrossRef Medline

Vullhorst D, Neddens J, Karavanova I, Tricoire L, Petralia RS, McBain CJ, Buonanno A (2009) Selective expression of ErbB4 in interneurons, but not pyramidal cells, of the rodent hippocampus. J Neurosci 29: 12255-12264. CrossRef Medline

Wang DD, Kriegstein AR (2008) GABA regulates excitatory synapse formation in the neocortex via NMDA receptor activation. J Neurosci 28: 5547-5558. CrossRef Medline

Witter MP (2007) The perforant path: projections from the entorhinal cortex to the dentate gyrus. Prog Brain Res 163:43-61. CrossRef Medline

Woo RS, Li XM, Tao Y, Carpenter-Hyland E, Huang YZ, Weber J, Neiswender H, Dong XP, Wu J, Gassmann M, Lai C, Xiong WC, Gao TM, Mei L (2007) Neuregulin-1 enhances depolarization-induced GABA release. Neuron 54:599-610. CrossRef Medline

Wu G, Malinow R, Cline HT (1996) Maturation of a central glutamatergic synapse. Science 274:972-976. CrossRef Medline

Yin DM, Chen YJ, Lu YS, Bean JC, Sathyamurthy A, Shen C, Liu X, Lin TW, Smith CA, Xiong WC, Mei L (2013) Reversal of behavioral deficits and synaptic dysfunction in mice overexpressing neuregulin 1 . Neuron 78 : 644-657. CrossRef Medline 